Draft VERSION OCTOBER 9, 2018

Preprint typeset using $\mathrm{LATEX}_{\mathrm{E}}$ style emulateapj v. 5/2/11

\title{
MAGNETO-THERMAL DISK WIND FROM PROTOPLANETARY DISKS
}

\author{
Xue-Ning Bai ${ }^{1}$, Jiani Ye², Jeremy Goodman ${ }^{3}$, Feng YuAn $^{2}$ \\ Draft version October 9, 2018
}

\begin{abstract}
Global evolution and dispersal of protoplanetary disks (PPDs) is governed by disk angular momentum transport and mass-loss processes. Recent numerical studies suggest that angular momentum transport in the inner region of PPDs is largely driven by magnetized disk wind, yet the wind mass-loss rate remains unconstrained. On the other hand, disk mass loss has conventionally been attributed to photoevaporation, where external heating on the disk surface drives a thermal wind. We unify the two scenarios by developing a 1D model of magnetized disk winds with a simple treatment of thermodynamics as a proxy for external heating. The wind properties largely depend on 1) the magnetic field strength at the wind base, characterized by the poloidal Alfvén speed $v_{A p}, 2$ ) the sound speed $c_{s}$ near the wind base, and 3) how rapidly poloidal field lines diverge (achieve $R^{-2}$ scaling). When $v_{A p} \gg c_{s}$, corotation is enforced near the wind base, resulting in centrifugal acceleration. Otherwise, the wind is accelerated mainly by the pressure of the toroidal magnetic field. In both cases, the dominant role played by magnetic forces likely yields wind outflow rates that well exceed purely hydrodynamical mechanisms. For typical PPD accretion-rate and wind-launching conditions, we expect $v_{A p}$ to be comparable to $c_{s}$ at the wind base. The resulting wind is heavily loaded, with total wind mass loss rate likely reaching a considerable fraction of wind-driven accretion rate. Implications for modeling global disk evolution and planet formation are also discussed.
\end{abstract}

Subject headings: accretion, accretion disks - magnetohydrodynamics — methods: numerical planetary systems: protoplanetary disks

\section{INTRODUCTION}

The theory of magnetized disk winds has been developed for decades, following the seminal work of Blandford \& Payne (1982, hereafter BP). Assuming a razor-thin (cold) disk and ideal MHD, poloidal magnetic field lines anchored to the disk behave as rigid wires and will centrifugally accelerate gas if they are inclined by more than $30^{\circ}$ to the rotation axis. The wind exerts a torque on the disk surface that efficiently extracts angular momentum and drives accretion. BP's analytical theory was subsequently generalized and refined to better account for disk structure and boundary conditions, and has been applied to jets and outflows from general accretion disks and young stellar objects (e.g., Pudritz \& Norman 1983, 1986; Konigl 1989; Lovelace et al. 1991; Pelletier \& Pudritz 1992; Contopoulos \& Lovelace 1994; Ferreira \& Pelletier 1995; Li 1995, 1996; Ferreira 1997; Ostriker 1997; Vlahakis et al. 2000). Global magnetohydrodynamic (MHD) simulations, less restricted by simplifying assumptions such as self-similarity and time-independence, have been widely adopted for studying global wind structure and evolution, as well as the wind launching process and disk-wind connection (e.g., Ouyed et al. 1997; Ouyed \& Pudritz 1997a,b, 1999; Ustyugova et al.

\footnotetext{
xbai@cfa.harvard.edu

${ }^{1}$ Institute for Theory and Computation, HarvardSmithsonian Center for Astrophysics, 60 Garden St., MS-51, Cambridge, MA 02138

${ }^{2}$ Key Laboratory for Research in Galaxies and Cosmology, Shanghai Astronomical Observatory, Chinese Academy of Sciences, 80 Nandan Road, Shanghai 200030, China

${ }^{3}$ Department of Astrophysical Sciences, Peyton Hall, Princeton University, Princeton, NJ 08544
}

1999; Krasnopolsky et al. 1999, 2003; Kato et al. 2002; Fendt \& Čemeljić 2002; Casse \& Keppens 2002, 2004; Anderson et al. 2005; Pudritz et al. 2006; Zanni et al. 2007; Tzeferacos et al. 2009; Porth \& Fendt 2010; Ramsey \& Clarke 2011; Tzeferacos et al. 2013). It is now well known that the wind properties depend mainly on the strength and distribution of the magnetic flux threading the disk, and on the mass loading, with the latter mainly controlled by disk physics and thermodynamics.

In this paper, we focus on MHD disk winds from very weakly ionized protoplanetary disks (PPDs). Due to low temperatures, PPDs are generally extremely weakly ionized, so that gas and magnetic fields are poorly coupled. Except near the star, the coupling depends upon external ionization sources such as cosmic-rays, X-rays, and UV photons (e.g., Hayashi 1981; Igea \& Glassgold 1999; Perez-Becker \& Chiang 2011) rather than ionization in local thermodynamic equilibrium. These sources have finite penetration depths and produce ionization levels that decrease from the disk surface toward the midplane.

It has recently been realized that in the inner region of PPDs $(\lesssim 10 \mathrm{AU})$, the magnetorotational instability (MRI, Balbus \& Hawley 1991), heretofore supposed to be the main driver of disk accretion, is almost completely suppressed (Bai \& Stone 2013b). This is mainly because of non-ideal MHD effects. Ohmic resistivity, the Hall effect, and ambipolar diffusion substantially reduce the coupling between gas and magnetic fields in weakly ionized gas. The coupling is in any case too poor for the disk to generate its own field via a self-contained MRI dynamo (Fleming et al. 2000; Oishi \& Mac Low 2011; Lesur et al. 2014; Riols et al. 2015); therefore, models for MRI-driven turbulence in PPDs posit net poloidal 
flux threading the disk. Even so, earlier works considering only Ohmic resistivity expected the MRI to operate near the disk surface $(1-2$ scale heights above midplane, e.g., Gammie 1996, see also Armitage 2011 for a review). But ambipolar diffusion completely changes the picture. Instead of turbulent MRI, the poloidal flux naturally launches a magnetized disk wind (Bai \& Stone 2013b; Bai 2013; Gressel et al. 2015, and see Turner et al. 2014 for a review). While inclusion of the Hall effect introduces further complications (Lesur et al. 2014; Bai 2014, 2015; Simon et al. 2015), it appears unavoidable that in the inner regions of PPDs, accretion is largely winddriven.

The PPD simulations of Bai \& Stone (2013b) and followup works cited above have demonstrated the robustness of MRI suppression and wind launching. However, the local (shearing-box) approximations used in these simulations do not permit the mass-loss and torque of the wind to be reliably determined. In particular, the location of the Alfvén point and mass loss rate depends on the vertical box size. Recent simulations by Gressel et al. (2015) were global in radius, but have the same vertical extent as earlier local simulations, and hence should be considered local in this sense.

While the aforementioned global studies of MHD disk winds considered PPDs to be among the primary applications, they generally failed to take into account realistic disk microphysics. Furthermore, the range of parameters considered in these studies is not appropriate for most PPDs, as we elaborate below.

Early analytical wind studies and simulations largely ignored disk microphysics. While more recent global disk wind simulations take into account the vertical structure of the disk, they all rely on a prescribed effective magnetic diffusivity with somewhat arbitrary strength, anisotropy and spatial dependence. In reality, the diffusivity is due either to MRI turbulence (in well-ionized disks) and/or to non-ideal MHD effects (in PPDs), effects that are not captured by these prescriptions. For PPDs, local simulations that take into account the dominant non-ideal MHD effects indicate that the wind is launched from several scale heights above midplane, where far-UV radiation is able to penetrate (Bai \& Stone 2013b; Bai 2013, 2014; Gressel et al. 2015). This location is much higher, and consequently the ratio of the local gas density to that at the midplane is much lower, than all previous models assumed. ${ }^{4}$

The choice of wind parameters has usually also been unrealistic, at least for PPDs. Most previous studies considered very strong disk magnetization, with midplane strengths close to equipartition: i.e. $\beta_{0} \equiv$ $\left(8 \pi p_{\text {gas }} / B_{z}^{2}\right)_{\text {midplane }} \sim O(1)$, where $p_{\text {gas }}$ is the gas pressure and $B_{z}$ the vertical field. For standard PPD models, angular momentum transport by such strong field would be so rapid that the disk would be drained in $\sim 10^{3}$ years instead of typical PPD lifetime of a few Myrs (see Appendix A for more discussion) ${ }^{5}$. While

\footnotetext{
4 Semi-analytical works of Königl et al. (2010); Salmeron et al. (2011); Teitler (2011) also took into account all non-ideal MHD effects to study wind launching from PPDs, but they did not consider realistic ionization profile and arrived at different conclusions.

5 To achieve realistic wind-driven accretion rate, wind models constructed by Combet \& Ferreira (2008) have surface density or-
}

near-equipartition field has been suggested to be necessary for wind launching (e.g., Wardle \& Koenigl 1993; Ferreira \& Pelletier 1995; Ogilvie 2012, partly to suppress the MRI), more recent simulations have showed that with reasonable prescriptions for magnetic diffusivity, steady disk winds can be launched by much weaker fields $\left(\beta_{0} \sim 500\right)$, as long as the disk corona is strongly magnetized (Murphy et al. 2010; Sheikhnezami et al. 2012; Stepanovs \& Fendt 2014). The expected level of magnetization in PPDs in standard disk models is still lower, by several orders of magnitude (see Appendix A).

In addition, most previous MHD wind studies, especially simulations, adopteded the conventional "cold MHD wind" scenario, which ignores the thermodynamics of the wind. (Notable exceptions are Casse \& Ferreira 2000 and Tzeferacos et al. 2013). PPDs are quite different from most other types of accretion disks in that their thermodynamics is dominated by external heating from the central protostar. In particular, the highenergy stellar X-ray and UV radiation, which provides the main source of disk ionization, also serves as the dominant heating source at disk surface and the entire wind zone. Without considering magnetic fields, such heating is sufficiently strong to drive "photoevaporation" of PPDs, which has been considered as the dominant mechanism for PPD mass loss and dispersal (see recent review by Alexander et al. 2014, and also Section 5.2 for further discussions). So far, photoevaporation models (pure thermal wind) of PPD mass loss have been developed in parallel with cold MHD disk wind models without overlap. In reality, the nature of PPD wind is likely a marriage of both magnetic and thermal effects: $P P D$ wind is both hot and magnetically dominated.

It is not yet feasible for us to perform global wind simulations that combine magnetohydrodynamics, tensorial conductivities, chemical and ionization reactions, and accurate thermodynamics. Even if we were able to do so, it might be difficult to interpret the results because they could be affected by many factors. Our approach here is to develop a simple global wind model that captures the essential physics of wind launching, acceleration, and propagation. The model is an extension of the classic Weber \& Davis (1967) wind described by relatively few parameters. This allows us to explore the roles of magnetic and thermal effects easily, if not fully consistently, and to reconnoitre those regions of parameter space that deserve further and more accurate scrutiny. We also expect our model to serve as a useful framework for interpreting more realistic global PPD wind simulations in the future.

Our model is similar in spirit to that of (Kudoh \& Shibata 1997, hereafter KS97) in that we prescribe the poloidal magnetic geometry and solve for the flow, toroidal field, and critical points along poloidal lines under ideal MHD. Whereas KS97 were interested in applications to fast winds launched from $\sim 15 \mathrm{R}_{\odot}$, however, we are interested in slower winds launched from $\gtrsim 1 \mathrm{AU}$ where the midplane is essentially uncoupled to the field, so that the wind base lies several scale heights above the midplane where X-rays and UV penetrate (Appendix A). Also we explore a greater

ders of magnitude lower than standard disk models. 
range of poloidal magnetic geometries, described by two parameters rather than KS97's one. Some of our main conclusions are in agreement with theirs, such as the importance of toroidal magnetic pressure in lauching the wind.

This paper is organized as follows. In $\S 2$, we describe the motivation and physical picture underlying our wind model. We also describe the wind equations and parameters, some diagnostic quantities, and our numerical methods. In $\S 3$, after presenting some illustriative wind solutions, we identify the more consequential wind parameters and systematically explore their influence on wind properties within the range that we believe relevant to PPDs. In $\S 4$, we show that the important wind properties, such as mass loading and specific angular momentum, are insensitive to the remaining input parameters, justifying in part our simplified wind model. General implications and broader context are provided in $\S 5$, and a summary of our main results and conclusions in $\S 6$.

\section{A SIMPLIFIED MODEL OF DISK WIND FROM PPDS}

We consider the inner region of PPDs $(\lesssim 10 \mathrm{AU})$, and assume axisymmetry and steady state. This is reasonable because we expect the disk to be largely laminar under realistic conditions (Bai 2013, 2014). As long as the gas and magnetic field are well coupled in the wind zone (i.e., in the ideal MHD regime), then gas must flow along magnetic field lines, and the MHD equations reduce to a set of one-dimensional conservation laws (e.g., Mestel 1961; Spruit 1996, as we will summarize shortly). This is the basis of MHD wind theory.

In the inner parts of PPDs, the bulk of the gas is cold and extremely weakly ionized. Non-ideal MHD effects (particularly Ohmic resistivity and ambipolar diffusion) are strong within several scale heights of the midplane (e.g., Wardle 2007; Bai 2011; Mohanty et al. 2013). As a result, gas is very poorly coupled to magnetic fields, and does not follow field lines, so that an MHD wind can not be launched efficiently. On the other hand, FUV can almost fully ionize trace species such as carbon and sulfur in the disk upper atmosphere, down to a column density $\sim 0.01-0.1 \mathrm{~g} \mathrm{~cm}^{-2}$, providing ionization fractions $\gtrsim 10^{-4}$ (Perez-Becker \& Chiang 2011). At this ionization level, non-ideal MHD effects become relatively unimportant in wind dynamics (but perhaps not thermodynamics: e.g., Safier 1993; Garcia et al. 2001). Indeed, with a simplified prescription for FUV ionization, local simulations by Bai \& Stone (2013b) showed that the base of the wind ${ }^{6}$ generally coincides with the FUV ionization front. The same conclusion is also arrived from (radially) global simulations of Gressel et al. (2015).

The FUV radiation not only significantly enhances the ionization fraction, but also significantly heats the gas. The thermal structure of the disk surface is highly complex, with differing gas and dust temperatures, strong molecular and atomic line emission, and

6 Technically, this corresponds to the location where the gas velocity transitions from sub-Keplerian (below) to superKeplerian (above), marking the onset of centrifugal acceleration (Wardle \& Koenigl 1993). While the wind launching process starts from somewhat deeper layers where coupling between gas and magnetic fields is marginal, the wind base so defined marks a clear transition in the flow properties (see Section 4 of Bai \& Stone 2013b).

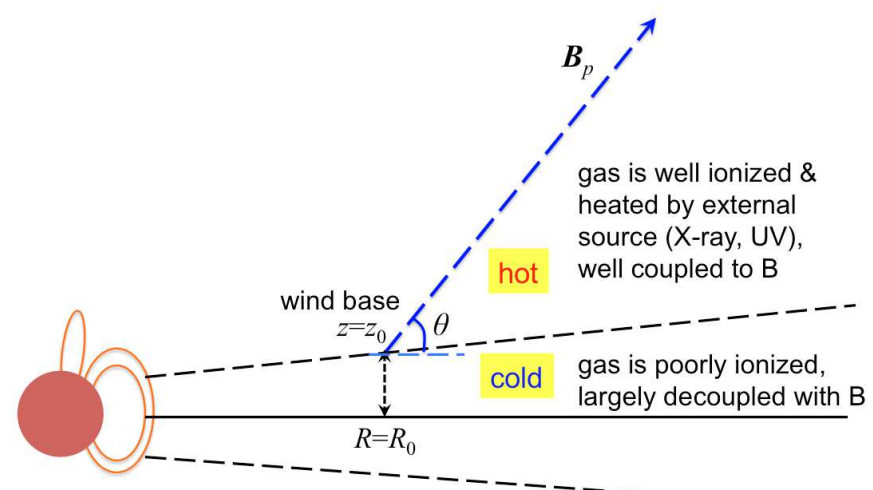

FIG. 1.- Schematic illustration of our simplified wind model. See Section 2.1 for details.

other non-LTE effects (see Bergin et al. 2007 for a review). It has been extensively studied in the framework of hydrostatic disk models (e.g., Glassgold et al. 2004; Nomura \& Millar 2005; Aikawa \& Nomura 2006; Woitke et al. 2009; Walsh et al. 2010; Bethell \& Bergin 2011; Fogel et al. 2011; Walsh et al. 2012; Akimkin et al. 2013). In general, the gas temperature increases dramatically from the cold disk through the CO photodissociation front and reaches a few thousand degrees in the FUV layer.

\subsection{Model Description}

Motivated by these discussions, we now describe a simplified magnetized model for PPDs as illustrated in Figure 1 . We use $R$ to denote cylindrical radius, and consider disk wind launched from a fiducial radius $R_{0}$. The disk is divided into a cold interior, where the gas is poorly coupled to magnetic fields and obeys hydrostatic equilibrium, and a hot exterior, where the gas is sufficiently well coupled and an MHD wind is launched. The transition occurs at vertical height $z=z_{0}$ from disk midplane, the location of the wind base. We prescribe the geometry of a typical poloidal field line anchored to the disk at the wind base $\left(R_{0}, z_{0}\right)$ and solve the conservation laws along this wind field line. For simplicity, poloidal field lines are taken to be straight, with a constant inclination angle $\theta$ relative to the equatorial plane at $z \geq z_{0}$. In parametric form, the field line is described by

$$
R(s)=R_{0}+s \cos \theta, \quad z(s)=z_{0}+s \sin \theta,
$$

where $s$ is the arc length along the poloidal field line.

In addition to poloidal field geometry, we also need to prescribe the functional form of $B_{p}(R)$. If the wind is launched from a radially extended region in an approximately self-similar manner, then the poloidal field would be approximately parallel near the wind launching region, and hence $B_{p}(R) \propto R^{-1}$. Towards large $R$, the solenoidal constraint demands $B_{p}(R) \propto R^{-2}$. (This is true of KS97's field, for example, although it collimates parabolically toward the axis, unlike ours.) We therefore assume the following functional form for $B_{p}(R)$ :

$$
B_{p}(R)=B_{p 0} \frac{1+q}{\left(R / R_{0}\right)+q\left(R / R_{0}\right)^{2}} .
$$

The parameter $q$ controls the radius at which the poloidal field lines transition from being parallel to diverging. 
Along the field line, let $\rho, \boldsymbol{v}_{p}$, and $v_{\phi}=\Omega R$ be gas density, poloidal velocity, and toroidal velocities, with $\Omega$ the gas angular velocity. Poloidal and toroidal magnetic fields are denoted $\boldsymbol{B}_{p}$ (prescribed) and $B_{\phi}$ (to be solved for). As noted earlier, the ideal MHD equations for a steady axisymmetric wind yield four constants of the flow. The first three of these are

$$
k \equiv \frac{4 \pi \rho v_{p}}{B_{p}}
$$

which follows from the continuity equation,

$$
\omega \equiv \Omega-\frac{k B_{\phi}}{4 \pi \rho R},
$$

which follows from the induction equation and can be interpreted as the angular velocity of the line, and the specific angular momentum

$$
l=\Omega R^{2}-\frac{R B_{\phi}}{k} .
$$

Thermodynamics is reflected in the energy equation via the enthalpy $h \equiv \int d P / \rho$. We assume that the gas in the wind zone can be approximately described by a barotropic equation of state $P=P(\rho)$ so that we simply have $h=h(\rho)$. By default, we choose the wind to be isothermal at sound speed $c_{\mathrm{s}, \mathrm{w}}$, so that $P=\rho c_{\mathrm{s}, \mathrm{w}}^{2}$ and

$$
h(\rho)=c_{\mathrm{s}, \mathrm{w}}^{2} \log \left(\rho / \rho_{0}\right),
$$

where $\rho_{0}$ is the density at the wind base. Energy conservation is then expressed via the Bernoulli constant

$$
E=\frac{v^{2}}{2}+h+\Phi-\omega R v_{\phi}=\frac{v_{p}^{2}+\left(v_{\phi}-\omega R\right)^{2}}{2}+h+\Phi_{\mathrm{eff}},
$$

where $v=\sqrt{v_{p}^{2}+v_{\phi}^{2}}$ is the total flow speed, $\Phi=$ $-G M_{*} / \sqrt{R^{2}+z^{2}}$ is the gravitational potential, and $\Phi_{\text {eff }}=\Phi-\frac{1}{2} \omega^{2} R^{2}$. Unlike "viscous" angular momentum transport (e.g., MRI turbulence), ideal MHD winds do not directly heat the disk. ${ }^{7}$

Although constant along each poloidal line, the four flow quantities $\{k, \omega, l, E\}$ vary between lines. Once we have prescribed the shape of a poloidal field line and its strength $B_{p}$ along the line, as well as the enthalpy $h(\rho)$, the wind solution is fully specified by the flow constants. The primitive flow variables $\left(\left\{\rho, v_{p}, v_{\phi}, B_{\phi}\right\}\right)$ are determined by $\{k, \omega, l, E\}$ via the defining equations (3)(7). However, not all of the flow constants can be chosen independently if the wind is to reach infinity with a terminal speed exceeding all of its characteristic velocities. The Bernoulli constant $E$ is essentially determined by physical conditions at the wind base. Also, one expects $\omega \approx \Omega_{K}\left(R_{0}\right)$, where $\Omega_{K}\left(R_{0}\right)$ is the Keplerian angular frequency at the wind base, and so we provisionally take $\omega=\Omega_{K}$. The remaining constants $k$ and $l$ are found by imposing regularity conditions at two critical points.

For a given disk model, therefore, the wind solution is determined by the following parameters:

${ }^{7}$ A low level of heating can be produced as magnetic flux drifts through the gas in the disk interior-and perhaps even within the wind (Safier 1993).
- Physical parameters: $B_{p 0}$ and $q\left[\right.$ eq. (2)] and $c_{\mathrm{s}, \mathrm{w}}$ [eq. (6)].

- Geometric parameters: base height $z_{0}$ and field inclination angle $\theta$.

We provide the expected range of these parameters and normalize them to dimensionless units in $\S 2.3$. The more general situation that $\omega \neq \Omega_{K}$ is discussed in $\S 4.2$.

Needless to say, our wind model involves substantial simplifications, especially with regard to the poloidal field and thermodynamics. In reality, the poloidal field geometry and strength are determined by force balance perpendicular to flux surfaces, as described by the GradShafranov equation (GSE), a nonlinear partial differential equation in $R$ and $z$. Besides the numerical difficulties involved in finding it, the solution to this equation depends on the global distribution of magnetic flux, which is largely unconstrained a priori. As a result, even when self-similarity is imposed, a diverse set of wind solutions is possible with vastly different field geometries. For instance, Ostriker (1997) showed that cylindrical collimation of a self-similar wind will not be achieved if the radial gradient of gas density and magnetic flux is steep, as is expected for PPDs.

Therefore, rather than solve the GSE, we have chosen to parametrize the poloidal field in terms of $B_{p 0}$ (its strength at the wind base), $\theta$ (inclination to the disk), and $q$ (divergence radius $q^{-1} R_{0}$ ). We expect the effects of more realistic field geometries to be roughly captured by systematically varying $\theta$ and $q$. Similarly, the role of external heating is encapsulated by an isothermal sound speed $c_{\mathrm{s}, \mathrm{w}}$, and by the wind-base height $z_{0}$, which reflects the penetration depth of external radiation. We also consider a polytropic equation of state in $\S 4.3$ to further test our simplified thermodynamics. In brief, despite the simplicity of our model, its parameters accomodate the essential physics of magnetized disk winds, and its simplicity facilitates the exploration and interpretion of their properties.

\subsection{Properties of Wind Equations and Critical Points}

It is convenient to define

$$
x \equiv \frac{4 \pi \rho}{k^{2}}=\frac{v_{A p}^{2}}{v_{p}^{2}},
$$

which rescales gas density $\rho$ and marks the ratio of the poloidal Alfvén velocity $v_{A p}=B_{p} / \sqrt{4 \pi \rho}$ to flow velocity. Eliminating $B_{\phi}$ from Equations (4) and (5) yields

$$
\omega-\Omega=\frac{l-\omega R^{2}}{(x-1) R^{2}}=\omega \frac{\left(R_{A} / R\right)^{2}-1}{x-1} .
$$

This relation defines the Alfvén point/radius $\left(R=R_{A}\right)$ : when the wind poloidal velocity reaches the Alfvén speed $(x=1)$, the numerator must also vanish, whence

$$
l=\omega R_{A}^{2} .
$$

The ratio $R_{A} / R_{0}$ is often called the magnetic lever arm, and $\left(R_{A} / R_{0}\right)^{2}$ is commonly denoted by $\lambda$ following BP. Although singular, the Alfvén point does not yield any 
additional constraint on the wind solution, and hence is not a critical point of the problem ${ }^{8}$.

Eliminating $v_{p}$ and $v_{\phi}=\Omega r$ from the energy equation (7) via eqs. (3) \& (9) yields

$$
\begin{aligned}
E & =\frac{1}{2} \frac{B_{p}^{2}}{k^{2} x^{2}}+\frac{\omega^{2} R^{2}}{2} \frac{\left(R_{A}^{2} / R^{2}-1\right)^{2}}{(x-1)^{2}}+h+\Phi_{\mathrm{eff}} \\
& \equiv H(x, s) .
\end{aligned}
$$

Once the values of $k, R_{A}, \& \omega$ are known, one can obtain $E=\left.H(x, s)\right|_{\rho=\rho_{0}, s=0}$ and then solve the algebraic equation $H(x, s)=E$ to obtain $x$ (or $\rho$ ) as a function of position along the line $(s$, or $R)$, thereby completing the wind solution.

For nonzero sound speed, however, additional constraints among $k, R_{A}, \omega$, and $E$ must be satisfied for a smooth solution. This can be seen from

$$
\begin{aligned}
x \frac{\partial H}{\partial x} & =-v_{p}^{2}-\frac{x}{x-1}(\Omega-\omega)^{2} R^{2}+c_{\mathrm{s}, \mathrm{w}}^{2} \\
& =\frac{v_{p}^{4}-\left(c_{\mathrm{s}, \mathrm{w}}^{2}+v_{A}^{2}\right) v_{p}^{2}+c_{\mathrm{s}, \mathrm{w}}^{2} v_{A p}^{2}}{v_{A p}^{2}-v_{p}^{2}},
\end{aligned}
$$

where $v_{A}=\sqrt{\left(B_{p}^{2}+B_{\phi}^{2}\right) / 4 \pi \rho}$ is the total Alfvén velocity. Clearly, the numerator becomes zero when the poloidal wind velocity $v_{p}$ equals the poloidal component of the slow or fast magnetosonic speed $\left(v_{s p}\right.$ or $\left.v_{f p}\right)$,

$$
v_{s p}^{2}, v_{f p}^{2}=\frac{\left(c_{\mathrm{s}, \mathrm{w}}^{2}+v_{A}^{2}\right) \mp \sqrt{\left(c_{\mathrm{s}, \mathrm{w}}^{2}+v_{A}^{2}\right)^{2}-4 c_{\mathrm{s}, \mathrm{w}}^{4}}}{2} .
$$

Correspondingly, $\partial H / \partial x=0$ defines the slow and fast magnetosonic points. In general, the algebraic equation $H(x, s)=E$ either has no solution for $x$ at each $s$ or two disconnected branches of solutions. A smooth wind solution is possible only when different branches join at the slow and fast magnetosonic points. At each of these two critical points, the following relations must be satisfied:

$$
H(x, s)=E, \quad \frac{\partial H(x, s)}{\partial x}=0, \quad \frac{\partial H(x, s)}{\partial s}=0 .
$$

Since these are three equations for two variables, a constraint results at each critical point, thereby determining $k$ and $R_{A}$ (equivalently, $k \& l$ ) in terms of $\omega \& E$.

In sum, to obtain the wind solution, the three algebraic equations (14) must be solved at both slow and fast magnetosonic points, which yield the location (radius) and density of the two critical points $\left(R_{s}, x_{s}, R_{f}, x_{f}\right)$, together with mass flux $k$ and Alfvén radius $R_{A}$. Energy flux is implicitly determined from $E=\left.H(x, s)\right|_{\rho=\rho_{0}, s=0}$.

These equations apply to any axisymmetric, steadystate, ideal-MHD wind. For instance, Weber \& Davis (1967)'s classic model is the special case $\{\theta=0, q=$ $\left.\infty, z_{0}=0\right\}$. Our wind solutions share many similarities with theirs. But with their inclined field geometry and launching from the disk surface, our winds experience different $\Phi_{\text {eff }}$, leading to different energetics.

8 The Alfvén point does become a critical point in solving crossfield balance via the GSE (e.g. BP, Spruit 1996).

\subsection{Dimensionless Units and Model Parameters}

In our calculations, we normalize lengths, velocities, and gas densities by their values at the footpoint of the field-line on the disk (wind base), so that $R_{0}=v_{K}=$ $\rho_{0}=1$. Correspondingly, time is in units of $1 / \Omega_{K}$.

Poloidal field strength is conveniently parameterized by $v_{A 0} \equiv B_{p 0} / \sqrt{4 \pi \rho_{0}}$, the poloidal Alfvén velocity at the footpoint. Appendix A discusses the expected parameter values for winds launched from $R_{0} \sim 1 \mathrm{AU}$. Based on that discussion, we choose the following fiducial parameters:

$$
\begin{aligned}
& v_{A 0}=c_{\mathrm{s}, \mathrm{w}}=0.1 v_{K}, \\
& z_{0}=0.15 R_{0}, \quad \theta=45^{\circ},
\end{aligned}
$$

with $\omega=\Omega_{K}$. We take $q=0.25$ as our fiducial choice for the functional form of $B_{p}$ in Equation (2), which means that transition from $R^{-1}$ (parallel) to $R^{-2}$ (diverging) occurs at around $R \sim 2 R_{0}$.

We find that $q, v_{A 0}$ and $c_{\mathrm{s}, \mathrm{w}}$ are the more important model parameters and focus on the dependence of wind solutions on these parameters in $\S 3$. We vary $v_{A 0} / v_{K}$ in the range between 0.01 and 10 , and $c_{\mathrm{s}, \mathrm{w}} / v_{K}$ between 0.03 and 0.3 . When exploring the role of poloidal field strength, only $v_{A 0}$ needs to be varied. However, when exploring the role of wind temperature at fixed $B_{p 0}$, in addition to varying $c_{\mathrm{s}, \mathrm{w}}$, one must also vary $v_{A 0}$ proportionally. This is because at fixed pressure (at wind base), the density scales inversely with temperature, thus affecting the Alfvén speed. For the field line divergence parameter $q$, we also consider $q=0.1$ (where transition occurs at $\left.\sim 3 R_{0}\right)$ and $q=\infty$ (purely diverging).

The dependence on the remaining parameters $\left(\theta, z_{0}\right.$, $\omega / \Omega_{K}$, equation of state) is explored in $\S 4$ and found to be relatively weak.

\subsection{Diagnostics of Wind Kinematics}

The most important wind diagnostic is the Alfvén radius $R_{A}$ (i.e. $R_{A} / R_{0}$ ): this characterizes the efficiency of the wind for extracting disk angular momentum. Since $\omega \approx \Omega_{K}$, the excess angular momentum per unit mass in the wind is $\Omega_{K}\left(R_{A}^{2}-R_{0}^{2}\right)$. The removal of this excess from the disk leads to a direct relation between the wind mass loss rate and wind-driven accretion rate.

Let $\dot{M}_{\text {acc }}$ be the wind-driven accretion rate at radius $R_{0}$. Angular-momentum conservation leads to

$$
\dot{M}_{\mathrm{acc}} \frac{d j}{d R}=\frac{d \dot{M}_{\mathrm{wind}}}{d R} \Omega_{K}\left(R_{A}^{2}-R_{0}^{2}\right)
$$

where $j(R) \equiv \Omega_{K} R^{2}$ is the specific angular momentum in the disk, and $\dot{M}_{\text {wind }}(R)$ denotes the cumulative mass loss rate from the origin to disk radius $R$. Noting that $d \ln j / d \ln R=1 / 2$ in Keplerian disks, we obtain

$$
\left.\xi \equiv \frac{d \dot{M}_{\text {wind }} / d \ln R}{\dot{M}_{\text {acc }}}\right|_{R=R_{0}}=\frac{1}{2} \frac{1}{\left(R_{A} / R_{0}\right)^{2}-1} .
$$

Ferreira \& Pelletier (1995) call $\xi$ the "ejection index."

Another important diagnostic quantity is the dimensionless "mass loading" parameter:

$$
\mu \equiv \frac{\omega R_{0}}{B_{p 0}} k=\frac{\omega R_{0} v_{p 0}}{v_{A 0}^{2}} .
$$



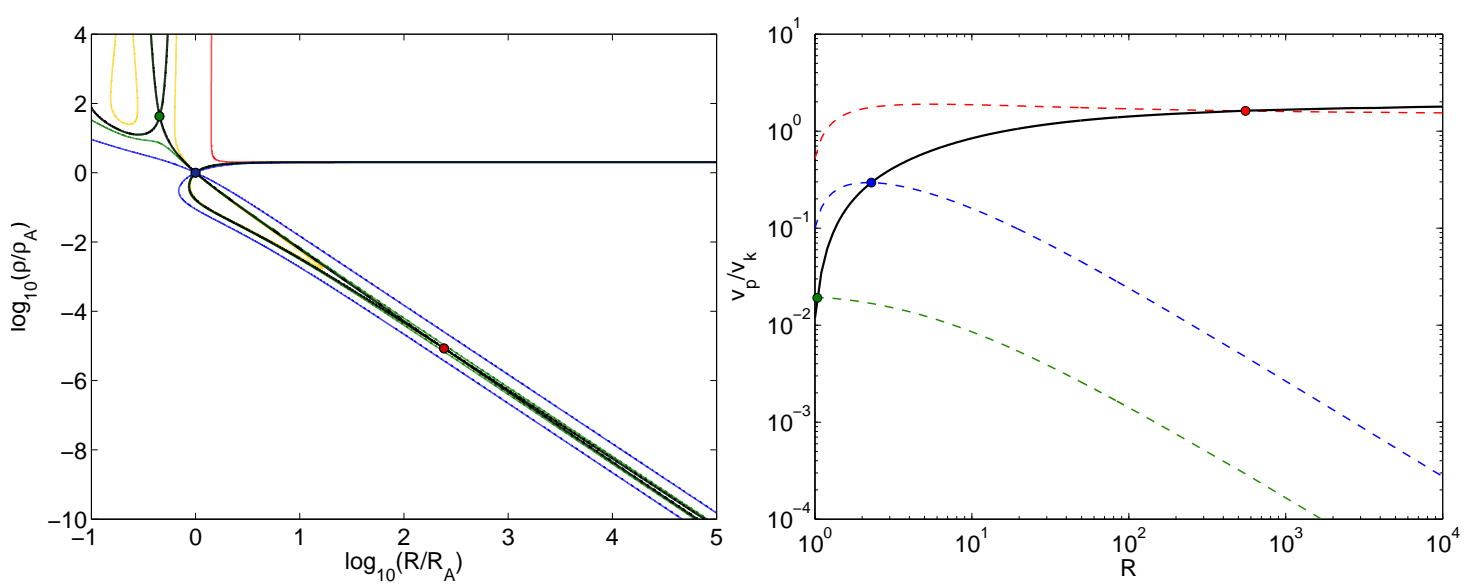

FIG. 2.- Representative wind solution for our fiducial parameters $q=0.25, v_{A 0}=c_{\mathrm{S}, \mathrm{w}}=0.1 v_{K}, \omega=\Omega_{K}, \theta=45^{\circ}, z_{0}=0.15 R_{0}$. Left: contours of the energy function $H(\rho, R)$. Black solid lines correspond to $H=E$, the Bernoulli constant, for the solution whose branches connect smoothly at both critical points. Yellow and red contours correspond to $H=E-0.2 v_{K}^{2}$, and $H=E-4 v_{K}^{2}$, respectively; green and blue contours to $H=E+0.2 v_{K}^{2}$, and $H=E+4 v_{K}^{2}$. Right: poloidal velocity profile of the wind solution (solid), and the green, blue and red dashed lines mark the poloidal components of slow magnetosonic, Alfvén and fast magnetosonic velocities. In both panels, green, blue and red dots mark the location of the slow, Alfvén and fast points.

A wind is considered "heavily loaded" if $\mu \gtrsim 1$, and "lightly loaded" if $\mu \ll 1$.

Mass accretion is due to the magnetic torque exerted at the disk surface/wind base (e.g. Bai \& Goodman 2009)

$$
\dot{M}_{\text {acc }}=\frac{2 R_{0}}{\Omega_{K}}\left|B_{z} B_{\phi}\right|_{z_{0}}=\frac{2 R_{0}}{\Omega_{K}} B_{p 0}^{2}\left|\frac{B_{\phi}}{B_{p}}\right|_{z_{0}} \sin \theta .
$$

The mass loss rate per logarithmic radius

$$
\frac{d \dot{M}_{\text {wind }}}{d \ln R}=2 \pi R^{2}\left(\rho v_{p}\right)_{z_{0}}=2 \pi R^{2} \rho_{0} v_{p 0},
$$

can be rewritten using (18) as

$$
\frac{d \dot{M}_{\text {wind }}}{d \ln R}=\frac{2 \pi R_{0}}{\omega} \mu \rho_{0} v_{A 0}^{2}=\frac{R_{0}}{2 \omega} \mu B_{p 0}^{2} .
$$

We see that accretion rate and mass loss rate have a common scaling factor $R_{0} B_{p 0}^{2}$. With $\omega \approx \Omega_{K}$, their ratio is then determined by the values of $\left|B_{\phi} / B_{p}\right|_{z 0}$ and $\mu$, which are determined by the wind solution. ${ }^{9}$

Other wind diagnostics are also of interest. One is the pitch of the field at the Alfvén point,

$$
\left.\frac{-B_{\phi}}{B_{p}}\right|_{R_{A}}=\left.\frac{\omega R_{A}}{v_{A p}}\left(-\frac{d \ln \rho}{d \ln R}\right)\right|_{R_{A}} ^{-1},
$$

which is a useful indicator of wind acceleration mechanism. Another is the wind terminal velocity $v_{p}^{\text {inf }}$, which relate the wind momentum and kinetic energy flux. A third is the ratio of Poynting flux to kinetic energy flux $\sigma$, which measures the efficiency of conversion of magnetic to kinetic energy. From Equations (3) and (4), it is straightforward to show that the Poynting flux is

9 Strictly, eq. (19) assumes that the wind base occurs where $v_{\phi}=\Omega_{K} R_{0}$ (Wardle \& Koenigl 1993). In our model, since $v_{\phi}$ is obtained from the wind solution, it does not strictly equal $\Omega_{K} R_{0}$ at our wind base. However, in practice the deviation is typically very small, and the ratio of (19) to (21) agrees with (17) within $10 \%$. In (vertically-local) simulations (Bai \& Stone 2013b; Gressel et al. 2015), the wind base as defined by WK93 coincides closely with the FUV ionization front (which we adopt as the wind base). $-\omega R B_{p} B_{\phi} / 4 \pi$. At $R \gg R_{A}$, we expect $\Omega \sim \omega R_{A}^{2} / R^{2} \ll$ $\omega$, hence from Equations $(4)$, we have $B_{\phi} \approx B_{p}\left(\omega R / v_{p}\right)$. With these relations, we obtain

$$
\sigma=\frac{-\omega R B_{p} B_{\phi}}{2 \pi \rho v_{p}^{3}} \approx \frac{B_{\phi}^{2}}{2 \pi \rho v_{p}^{2}} \approx 2 \frac{v_{A}^{2}}{v_{p}^{2}} .
$$

We note that because our wind solution passes the fast magnetosonic point, and $v_{f p}>v_{A}$, the asymptotic value of $\sigma$ at infinity must be smaller than 2 .

For a cold wind $\left(c_{s} \sim 0\right)$ in the Weber \& Davis wind model $(\theta=0, q=\infty)$, Spruit (1996) showed that the slow and fast magnetosonic points are located at the wind footpoint and infinity, respectively, and obtained analytical wind solutions. In particular, a very useful relation derives from the fast-point condition:

$$
\left(\frac{R_{A}}{R_{0}}\right)^{2}=\frac{3}{2}\left(1+\mu^{-2 / 3}\right) .
$$

This scaling relation has been commonly observed in simulations cold MHD disk winds (Ouyed \& Pudritz 1997a; Anderson et al. 2005; Zanni et al. 2007). Another useful relation states that

$$
v_{p}^{\inf } /\left(\omega R_{0}\right)=\mu^{-1 / 3}
$$

Hence lightly loaded winds achieve higher terminal velocities, although limited by the weak $1 / 3$ power. Spruit also showed that at the Alfvén point,

$$
\left|\frac{B_{\phi}}{B_{p}}\right|_{R_{A}} \approx\left\{\begin{array}{ll}
(19 / 8)^{1 / 2} & (\mu \ll 1) \\
1.14 \mu & (\mu \gg 1)
\end{array} .\right.
$$

Hence heavily-loaded winds have tightly wrapped toroidal fields at the Alfvén point. We will test these relations in our more general wind models.

\subsection{Numerical Method}

We solve the six algebraic equations in Equations (14) by a series of numerical iterations. In brief, we start from initial guesses for $R_{A}$ and $k$ and solve for the locations 

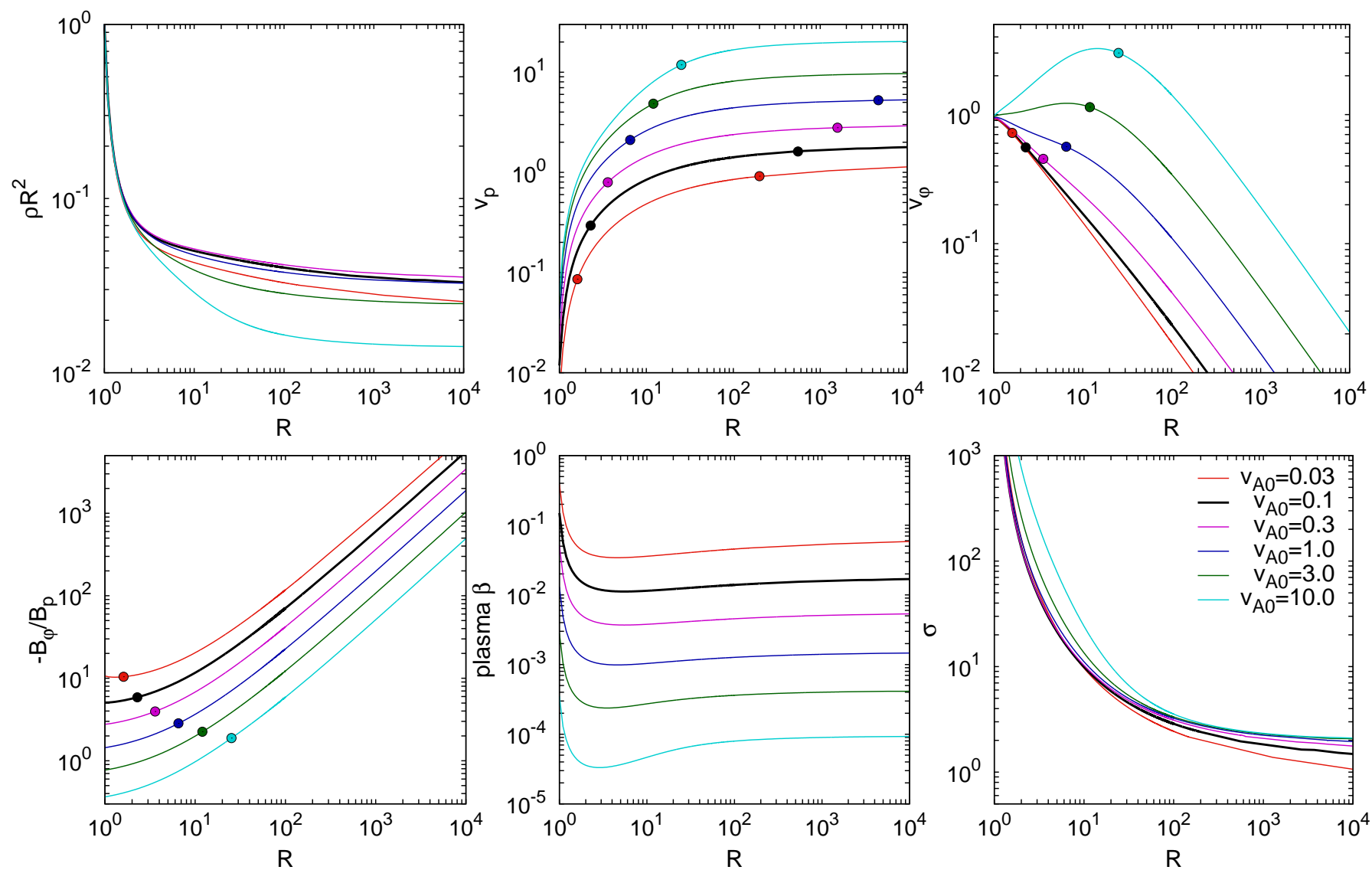

FIG. 3.- Profiles of various physical quantities along poloidal field lines as functions of cylindrical radius $R$. We vary field strength $v_{A 0}$ (see legend) while fixing $c_{\mathrm{s}, \mathrm{w}}=0.1 v_{K}, q=0.25$. Bold black line corresponds to our fiducial set of parameters. Alfvén points of the solutions are marked by filled circles in three of the panels. Fast magnetosonic points are marked in top middle panel (but lie outside the box for two top curves). Bottom middle panel: $\beta$ is the ratio of gas pressure to magnetic pressure, computed from the total field. Bottom right panel: $\sigma$ is the ratio of Poynting flux to kinetic energy flux (eq. 23).

of the slow and fast magnetosonic points by $\partial H / \partial \ln x=$ $\partial H / \partial \ln R=0$. We analytically calculate the second derivatives of $H$ with respect to $x$ and $R$ to guide the root-finding routines. The next stage of iteration involves adjustments of $k$ and $R_{A}$ so that $H\left(x_{s}, R_{s}\right)=E$ and $H\left(x_{f}, R_{f}\right)=E$ are satisfied, via a version of Newton's method.

To illustrate the mathematical structure of a typical wind solution, we show in Figure 2 for our fiducial parameter set the contours of the energy function $H$ in the plane of $\rho / \rho_{A}$ and $R / R_{A}$. Clearly, the slow and fast critical points are saddle points of $H$. A smooth wind solution requires that these two saddle points lie at intersections of contours where $H=E$, the Bernoulli constant. The solution for $\rho$ (or $x$ ) as a function of $R$ (or $s$ ) smoothly connects the three singular points (slow, Alfvén and fast). Beyond the fast magnetosonic point, the solution takes the lower branch. The right panel of the Figure further illustrates that the flow is continuously accelerated, passes the three MHD wave velocities at the location of the three singular points, and achieves an asymptotic velocity that is close to the Keplerian velocity at the wind launching point.

While solving for the slow point and $k$ at fixed $R_{A}$ is straightforward, success in finding the fast point sometimes requires a shrewd initial guess. This is related to the fact that the two branches of the $H=E$ contour are almost parallel to each other near the fast point (Fig. 2).
We surmount this numerical difficulty by starting from a standard wind solution, and then gradually altering its parameters towards the desired set, using each solution as the initial guess for the next. Once $k$ and $R_{A}$ are found, it is straightforward to obtain the solution $x=x(s)$ by solving $H(x, s)=E$ either by iteration or by tracking the $H=E$ contours from the critical points.

In certain cases, the slow point is located below the wind base with $R_{s}<R_{0}$, a situation that violates our assumption that ideal MHD applies throughout the wind (including the critical points). When this occurs, our wind solution is inaccurate, and we mark it separately.

\section{REPRESENTATIVE WIND SOLUTIONS}

In this section, we present representative wind solutions where we fix the wind geometry at $\theta=45^{\circ}$ at $z_{0}=0.15 R_{0}$, and choose $\omega=\Omega_{K}$. We explore the role played by key parameters including field strength $\left(v_{A 0}\right)$, field divergence parameter $q$, and wind temperature $\left(c_{\mathrm{S}, \mathrm{w}}\right)$, varying them around our fiducial values $v_{A 0}=c_{\mathrm{S}, \mathrm{w}}=0.1 v_{K}$ and $q=0.25$.

\subsection{Role of Poloidal Field Strength}

We first fix $c_{\mathrm{s}, \mathrm{w}}=0.1$ and $q=0.25$, and explore the role of poloidal field strength by varying $v_{A 0} / v_{K}$ from 0.03 to 10 . In Figure 3, we show the radial profiles of various wind diagnostic quantities. 

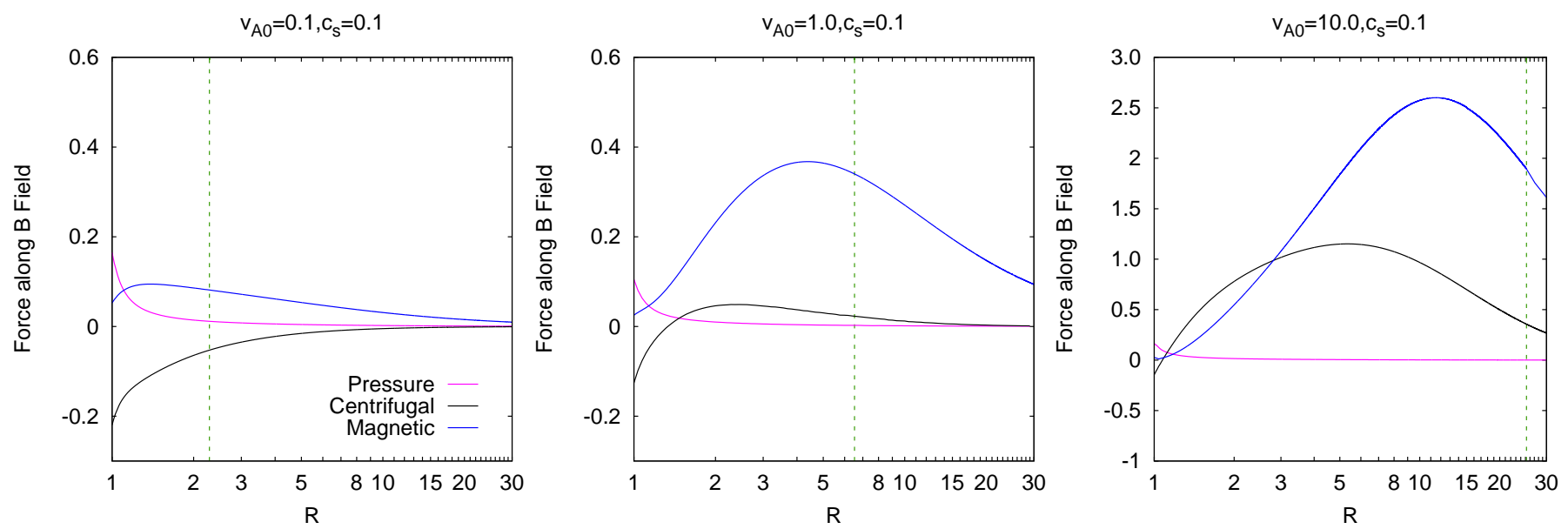

FIG. 4.- Wind acceleration mechanism for three of the solutions shown in Fig. (3) with $v_{A 0} / v_{K}=0.1,1.0$ and 10.0. Poloidal forces are decomposed into pressure-gradient, centrifugal, and magnetic according to eq. (27). Vertical dashed green lines mark the Alfvén radii.

All solutions accelerate monotonically from the windlaunching region, smoothly pass the critical points, and approach terminal velocities comparable to or beyond the Keplerian velocity at the wind base. Typically, near the wind base, the poloidal velocity is very small, and the gas is pressure supported, with significant contribution from magnetic pressure (see §3.1.1). In all solutions, the gas density decreases with $R$ (and hence $z$ ) as if in (magneto)hydrostatic equilibrium in this region. At larger radii, the wind achieves its asymptotic velocity $v_{p}^{\text {inf }}$, and hence the gas density scales in the same way as $B_{p}$, i.e. $R^{-2}$, while the scalings $B_{\phi} \propto R^{-1}$ and $v_{\phi} \propto R^{-1}$ follow directly from the conservation laws.

The Alfvén radius $R_{A}$ is a rapidly increasing function of $v_{A 0}$. Using a small $v_{A 0}=0.03 v_{K}$, we obtain $R_{A} \approx$ $1.61 R_{0}$. Such small $R_{A}$ would result in extremely efficient mass loss, with ejection index $\xi=\left(d \dot{M}_{w} / d \ln R\right) / \dot{M}_{a} \approx$ 0.4 (eq. 17). If the wind were launched over an extended region of the disk - say 0.3-15 AU as suggested by local disk simulations (Bai 2013) - the integrated wind mass loss rate (assuming $R_{A} / R_{0}$ roughly constant) would even exceed the accretion rate: $\dot{M}_{w} \approx 1.5 \dot{M}_{a}$ ! We defer the discussion of the possible consequences of such extreme mass loss to $\S 5$. On the other hand, increasing $v_{A 0}$ to values $\gtrsim v_{K}$ moves the Alfvén radius beyond $5 R_{0}$. In this limit, we have $\xi \lesssim 0.02$ and mass loss is almost negligible compared with accretion. Our fiducial solution $\left(v_{A 0}=c_{\mathrm{s}, \mathrm{w}}=0.1 v_{K}\right)$ has $R_{A} / R_{0} \approx 2.288$, gives a modest ejection index $\xi \approx 0.12$ which, if approximately constant over an extended radial range (e.g., again 0.3-15 AU), still yields a total mass loss rate that is a considerable fraction $(47 \%)$ of the wind-driven accretion rate.

For a cold $\left(c_{\mathrm{s}, \mathrm{w}} \ll v_{A}\right)$ wind, Ferreira (1997) derived the upper limit $\xi<0.15$ from energetic considerations. We are not restricted by this constraint because our wind has finite temperature. Our solutions violate this relation mainly when $v_{A 0} \lesssim c_{\mathrm{s}, \mathrm{w}}$, so that the wind is not cold in the launching region. Later in $\S 3.2$, we will see that over a wide range of parameter space, it is the ratio $v_{A 0} / c_{\mathrm{s}, \mathrm{w}}$ that largely sets the value of $R_{A}$ and hence $\xi$.

The fast magnetosonic points are generally located at very large distances. Although they are needed to find the Alfvén radii, the general wind properties are insen- sitive to the conditions at large distances (see $\S 4.3$ for an example). This is because the Bernoulli constant (7) is largely dominated by the kinetic energy terms there, while the enthalpy and gravitational potential terms become negligible.

In all cases, the wind is magnetically dominated throughout, as measured by plasma $\beta$, defined as the ratio of gas pressure to total magnetic pressure. Asymptotically, toroidal field wind up, giving $B_{\phi} / B_{p} \propto R$. At the wind base, toroidal field still dominates unless the system is very strongly magnetized with $v_{A 0} \gtrsim v_{K}$, as seen from the bottom left panel of Figure 3. This fact is directly related to the wind acceleration mechanism, as we discuss below in detail.

\subsubsection{Acceleration mechanism}

The wind acceleration process is best understood by directly considering the poloidal forces:

$$
\frac{d v_{p}}{d t}=\left[-\frac{d h}{d R}+\left(\frac{v_{\phi}^{2}}{R}-\frac{d \Phi}{d R}\right)-\frac{B_{\phi}}{4 \pi \rho R} \frac{d\left(R B_{\phi}\right)}{d R}\right] \frac{d R}{d s} .
$$

The first term in brackets is the thermal pressure gradient, and the last combines the tension and pressure gradient of the toroidal field.

We define the second term as the net centrifugal force (per unit mass), meaning the excess of centrifugal over gravitational acceleration. In the classical picture of magneto-centrifugal winds, a strong poloidal field dominates in the wind launching region. The stiffness of the poloidal field forces the flow to corotate with the wind footpoint (base) up to the Alfvén point, and accelerates the gas like "beads-on-a-wire." The net centrifugal force thus dominates the acceleration in this scenario.

In Figure 4, we show the force decomposition discussed above for three representative wind solutions shown in Figure 3 with different $v_{A 0}$. We see that when $v_{A 0}$ is small $\left(\sim 0.1 v_{K}\right)$, the net centrifugal force is always negative, and hence contributes negatively to the acceleration, which is almost entirely driven by the magnetic pressure gradient. The dominant role of magnetic pressure gradient in the case of weak poloidal field was also pointed out in KS97, and discussed in Lynden-Bell $(1996,2003)$. For larger $v_{A 0} \sim v_{K}$, the net centrifugal 
force is positive over a finite range of radii, yet acceleration is still dominated by magnetic forces. Only when $v_{A 0} \gg v_{K}$ does centrifugal acceleration dominate, although the dominance never extends to the Alfvén point within our range of parameters.

The effectiveness of centrifugal acceleration is directly related to how well co-rotation can be enforced. This can be seen from the top right panel of Figure 3: co-rotation is enforced only for $v_{A 0}=10 v_{K}$. For smaller $v_{A 0}$, the gas rotates substantially more slowly, and when $v_{A 0} \sim c_{\mathrm{s}, \mathrm{w}}$, $v_{\phi}$ begins almost immediately to drop as $1 / R$, suggesting little exchange of angular momentum with the field.

The lack of co-rotation is in line with the dominance of toroidal field discussed earlier. Enforced co-rotation only when the poloidal field is stiff enough to resist the tendency of the inertia of the gas to bend it into toroidal field. The fact that our wind is not "cold" makes magnetic field more prone to bending by increasing the mass loading.

In brief, our results suggest magnetic pressure gradient to plays a dominant role on wind launching in protoplanetary disks. In their original local study of PPD winds, Bai \& Stone (2013b) analyzed the local wind kinematics and concluded that wind acceleration is centrifugal. This apparent discrepancy is due to the different reference frames adopted. The local shearing box is formulated in a frame corotating with the disk, where the force equation becomes [see eq. (7) \& Spruit 1996]

$$
\frac{d v^{\prime 2}}{d s}=-\frac{d}{d s}\left(h+\Phi_{\mathrm{eff}}\right) \text {. }
$$

Here, $v^{\prime}$ is the total velocity in a frame corotating with the field line (angular velocity $\omega$ ), where its acceleration is mainly compensated by the drop in $\Phi_{\text {eff }}$. However, when corotation is not enforced, the change in $v^{\prime}$ mainly reflects the deviation of $v_{\phi}$ from $\omega R$ rather than acceleration of $v_{p}$. Indeed Bai \& Stone (2013b) pointed out that the wind launching process is largely due to the vertical magnetic pressure gradient of the toroidal field. Our results here show that this continues to be true of a global wind.

The fact that typical PPD winds are expected to be launched by magnetic pressure gradients raises concerns about stability. With toroidal field highly dominating poloidal field from the wind base, the wind velocity below the Alfvén point $v_{p}=v_{A p}$ is already much smaller than the total Alfvén velocity. For a single flux rope, kink instability is expected to develop on Alfvén-crossing timescales, although the situation is more complicated and less clear for disk winds with extended flux distributions (Spruit 1996). This question needs to be investigated via 3D MHD simulations. Previous 2D axisymmetric wind simulations also reported that no stable wind solution can be found for sufficiently low magnetization (e.g., Murphy et al. 2010; Sheikhnezami et al. 2012), although higher resolution and more realistic physical input are needed to clarify the threshold magnetization. Magnetic pressure driven outflows have also been identified in star formation simulations (Machida et al. 2008; Hennebelle \& Fromang 2008), and the kink instability appears to disturb the coherent toroidal field structure without affecting the acceleration (Tomida et al. 2013).
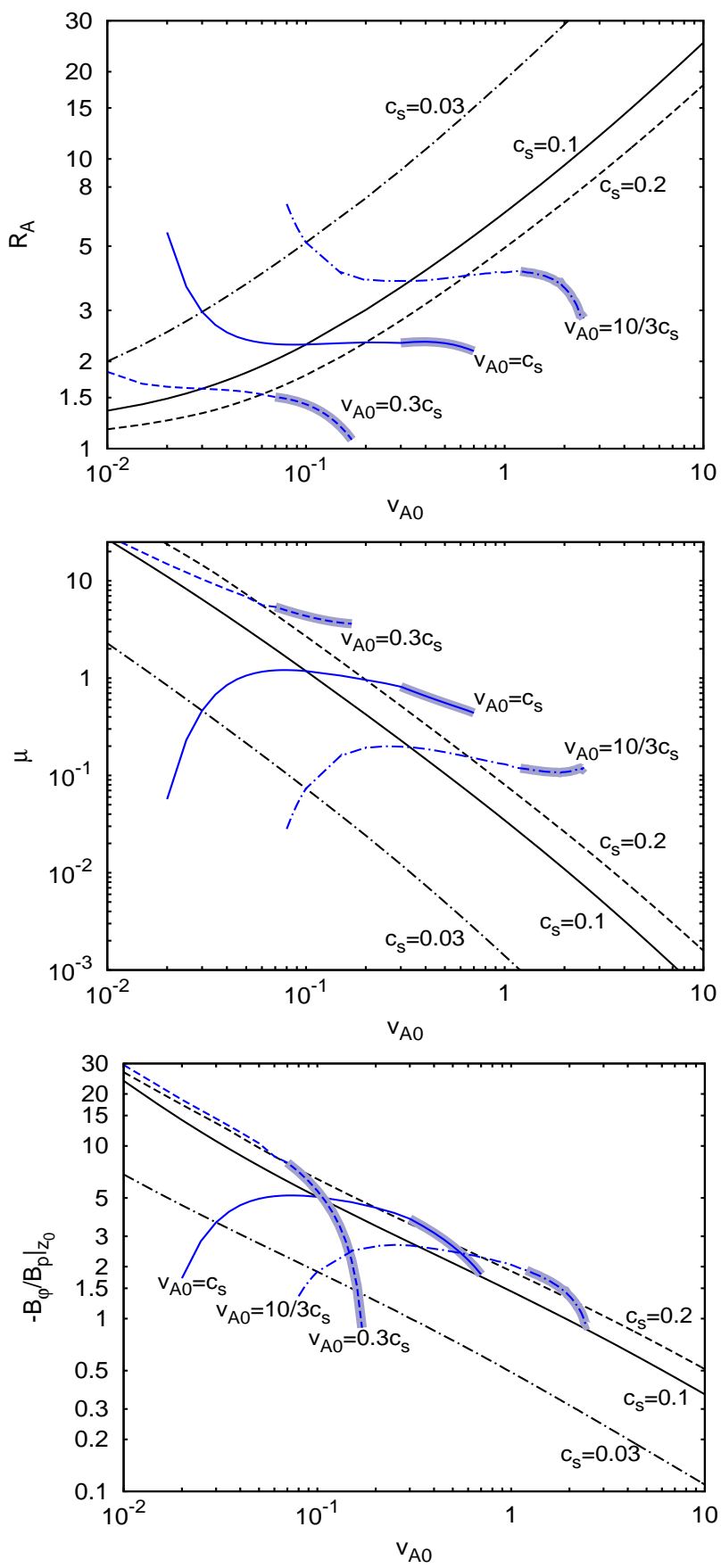

Fig. 5.- Dependence of Alfvén radius $R_{A}$ (top), mass loading $\mu$ (middle) and $\left|B_{\phi} / B_{p}\right|_{\text {base }}$ (bottom) on $v_{A 0}$ for different choices of $c_{\mathrm{S}, \mathrm{w}}$ (fixed or $\propto v_{A}$ ), as marked, with $q=0.25$ throughout. Shaded regions of blue lines have $R_{s}<R_{0}$, which violates our assumptions.

On the other hand, recent 3D disk wind simulations by Sheikhnezami \& Fendt (2015) with relatively strong magnetization found no sign of kink instability.

\subsection{Role of Wind Thermodynamics}

The balance among heating, radiative cooling, and adiabatic expansion affects the wind properties in two ways. First, it affects the internal wind temperature, which we represent by a constant $c_{\mathrm{s}, \mathrm{w}}$ (isothermal). We will further explore different equation of states in $\S 4.3$ and show 

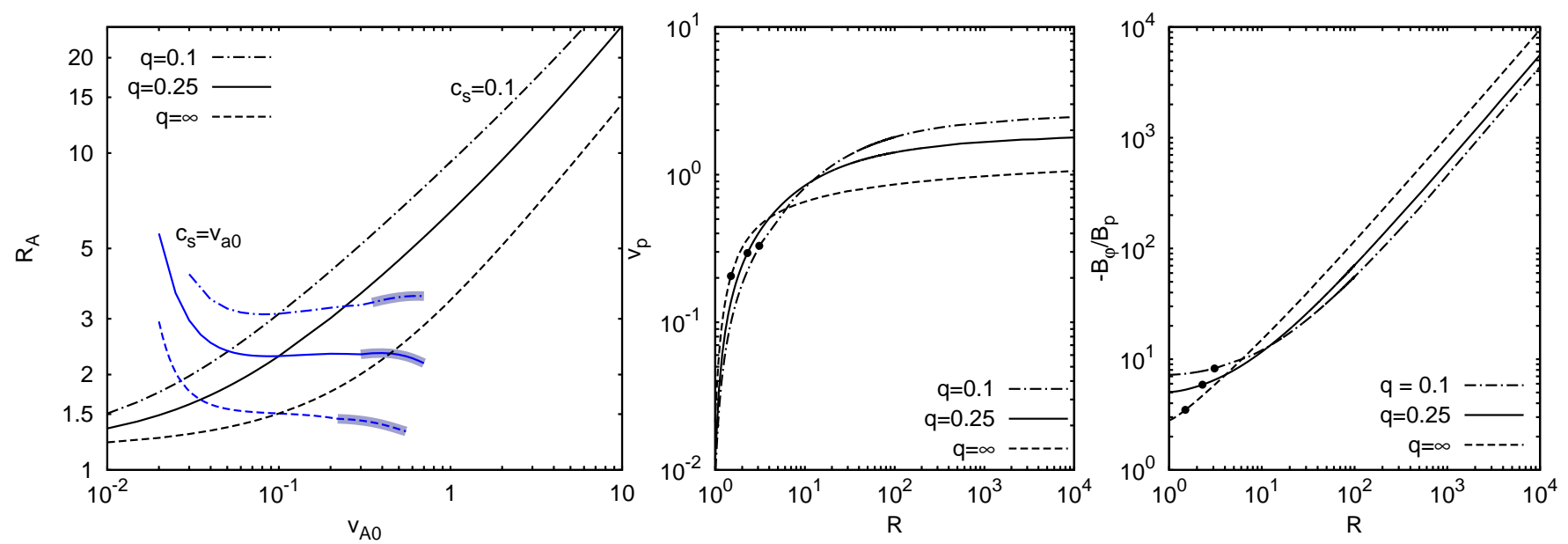

FIG. 6.- Left: Alfvén radius $R_{A}$ as a function of $v_{A 0}$ for different field divergences $q$ [eq.(2)] as marked in the legend. Black lines for fixed $c_{\mathrm{s}, \mathrm{w}}=0.1$, blue lines for $c_{\mathrm{s}, \mathrm{w}}=v_{A 0}$. Shaded portions have $R_{s}<R_{0}$, violating our assumptions. Middle: poloidal wind velocity along field lines for solutions with different $q$, as marked, and fixed $c_{\mathrm{s}, \mathrm{w}}=v_{A 0}=0.1$. Black dots mark Alfvén points. Right: similar to the middle panel, but for the field pitch $-B_{\phi} / B_{p}$.

that the additional effect is minor. Second, it affects the location of the wind base $z_{0}$. As discussed in Appendix $\mathrm{A}$, at fixed field strength, $v_{A 0}$ depends exponentially on $z_{0}$ : deeper penetration would reduce $v_{A 0}$. While we fix $z_{0}=0.15 R_{0}$ in this section, we show in $\S 4.1$ that the absolute value of $z_{0}$ is unimportant, thus the penetration depth affects the wind properties mainly by affecting $v_{A 0}$.

In the top panel of Figure 5, we fix $q=0.25$, and show the dependence of Alfvén radius on $v_{A 0}$ for two different values of $c_{\mathrm{s}, \mathrm{w}}$. According to our discussions above, black lines (fixed $c_{\mathrm{S}, \mathrm{w}}$, varying $v_{A 0}$ ) can be considered as varying the penetration depth at fixed wind temperature and field strength. Some consequences have been discussed in the previous subsection, but are now endowed with a new interpretation. Namely, enhanced penetration of external radiation would reduce the importance of magnetic fields, leading to a weaker wind with lower terminal velocities, smaller Alfvén radius, and more toroidally dominated (tightly wound) magnetic field.

The blue lines in the left panel correspond to varying the wind temperature at fixed penetration and field strength (see Section 2.3). We see that over a large range, these lines are relatively flat, suggesting that the location of $R_{A}$ is to a large extent controlled by the ratio of $v_{A 0} / c_{\mathrm{s}, \mathrm{w}}$ at the wind launching region. The contrast among these lines is very significant. Varying $v_{A 0} / c_{S}$ around unity by a factor of $\sim 3.3$ makes the $R_{A} / R_{0}$ vary from $\sim 2.3$ to $\sim 1.4$ and $\sim 4$, Because the ratio $\dot{M}_{w} / \dot{M}_{a}$ depends quadratically on $R_{A} / R_{0}$, such variations in the latter would easily give variations in wind mass loss rate by factors $\sim 10$. This discussion best demonstrates the sensitive dependence of the wind mass loss rate on the thermodynamics.

In reality, stronger external radiation is likely to lead to both higher wind temperature and deeper penetration. Therefore, enhancing external radiation may correspond to moving in between rightward (larger $c_{\mathrm{s}, \mathrm{w}}$ ) and to the bottom left (deeper penetration) in the top panel of Figure 5, along directions between tangents to the blue and black curves.

The middle and bottom panels of Figure 5 further show the dependence of the mass loading parameter $\mu$ and toroidal-to-poloidal field ratio at the wind base $\left|B_{\phi} / B_{p}\right|_{z_{0}}$. They are directly relevant to calculate the wind-driven accretion and wind mass loss rate via eqs. (19) \& (21).

Similar to the discussions above, increasing the penetration depth at fixed temperature (black lines, towards smaller $v_{A 0}$ ) yields both larger mass loading, and larger $\left|B_{\phi} / B_{p}\right|$ at wind base. The increase in $\mu$ is in fact faster, leading to higher ratio of mass loss rate over wind-driven accretion rate, in accordance with the trend seen for $R_{A}$ in the top panel. Note again that this discussion applies equally to varying poloidal field strength at fixed temperature. Increasing the temperature at fixed poloidal field strength (blue lines, towards larger $v_{A 0}$ ) yields similar trend in both $\mu$ and $\left|B_{\phi} / B_{p}\right|_{z_{0}}$ (ignoring the shaded portion of the lines), consistent with the top panel where the blue lines are largely horizontal over a wide range of $v_{A 0}$.

\subsection{Role of Field Line Divergence}

The field line divergence parameter $q$ characterizes the location $R_{t} \approx q^{-1 / 2} R_{0}$ where field line geometry transitions from being approximately parallel to diverging. Besides the fiducial value $q=0.25$, we explore two additional cases with $q=0.1$ and $q=\infty$. The main results are shown in Figure 6.

One sees that the Alfvén radius increases as $q$ decreases. Note that at fixed $B_{p 0}\left(\right.$ or $\left.v_{A 0}\right)$, smaller $q$ yields a stronger $B_{p}$ at $R>R_{0}$. Therefore, the trend of $R_{A}$ with $q$ is consistent with expectations from $\S 3$.1. In addition to larger $R_{A}$, smaller $q$ values yield higher terminal speeds, and less tightly wound fields, as shown in the middle and right panels. It is also interesting to note that the wind field is more toroidally dominated at the wind base for smaller (rather than larger) $q$, but the trend reverses at larger $R$. Therefore, smaller $q$ makes the magnetic pressure gradient more dominant in accelerating the wind.

Given the field strength and wind temperature, a lower limit of $R_{A}$ is set by $q=\infty$, where field lines diverge from the beginning. For $v_{A 0}=c_{\mathrm{s}, \mathrm{w}}=0.1 v_{K}$, we obtain $R_{A} \approx 1.51$, as opposed to $R_{A}=2.29$ when $q=0.25$. 

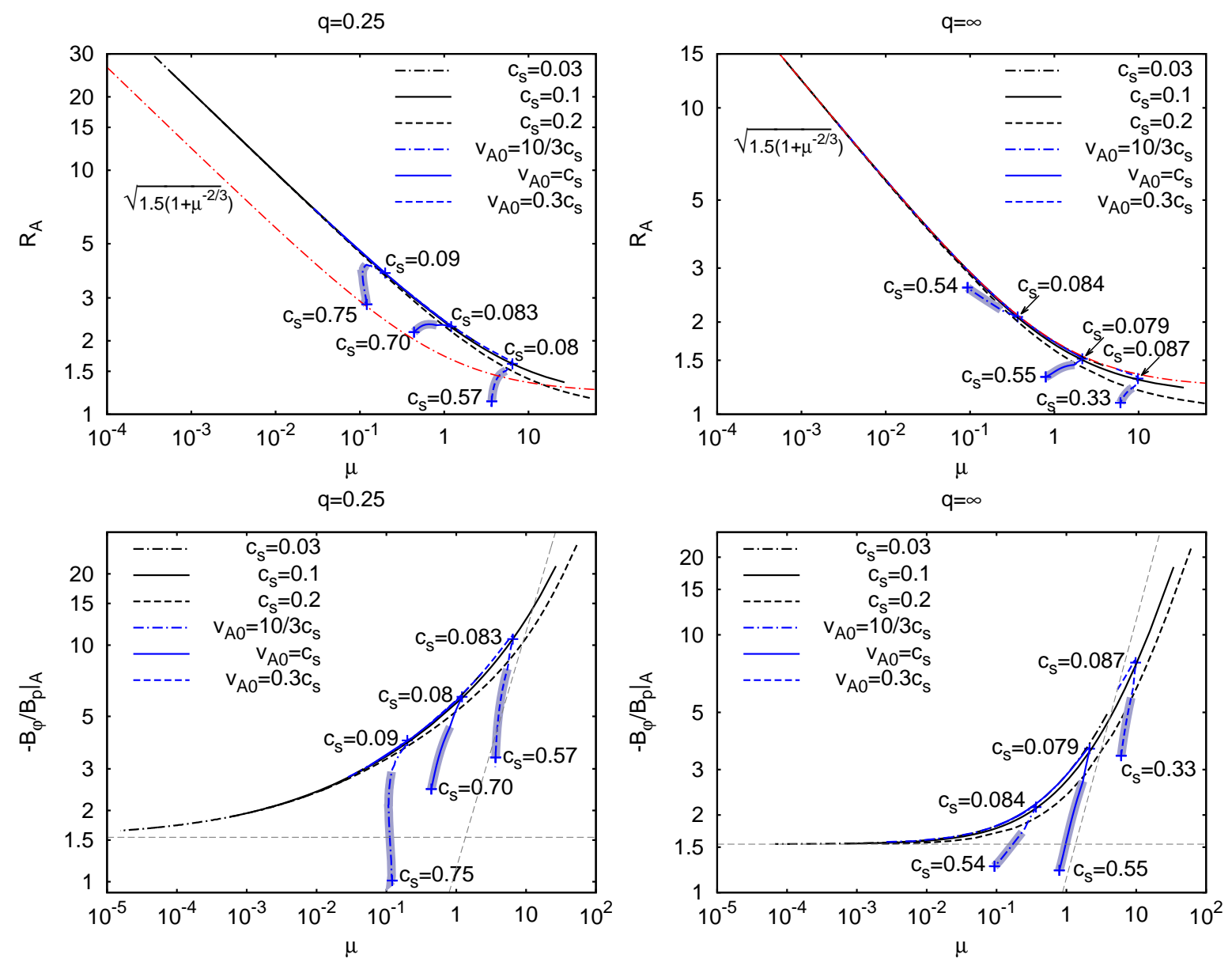

FIG. 7. - Scaling relations between pairs of wind diagnostics. The top two panels show the relation between the mass loading parameter $\mu$ and $R_{A}$, while the bottom two panels show the scaling relation between $\mu$ and $\left.\left(-B_{\phi} / B_{p}\right)\right|_{R_{A}}$. Left (right) panels correspond to wind solutions with $q=0.25(q=\infty)$. In each panel, we either choose fixed $c_{\mathrm{s}, \mathrm{w}}$, or choose $c_{\mathrm{s}, \mathrm{w}}$ to be proportional to $v_{A 0}$, as marked in the legends. To guide the eye, we further label the value of $c_{\mathrm{S}, \mathrm{w}}$ at various turning and tip points in the blue lines. The red dash-dotted line in the top panels indicates the expected scaling relation for the cold Weber \& Davis wind model. Similarly, the gray dashed lines in the bottom panels are the expected asymptotic scaling relations. Shaded regions have solutions with $R_{s}<R_{0}$, which would violate our assumptions, but are still shown for reference.

Our choice of $q=0.1$ corresponds to $R_{t} \sim 3 R_{0}$, which is already sufficiently far from the wind launching region. ${ }^{10}$ Therefore, this case may be considered to set an upper limit to $R_{A}$, giving $R_{A} \approx 3.11$ under the same conditions.

Overall, we see that even at fixed $v_{A 0}$ and $c_{\mathrm{s}, \mathrm{w}}$, the possible values of $R_{A}$ span a wide range depending on how rapidly poloidal field lines diverge, leading to variations in $\xi=\left(d \log \dot{M}_{w} / d \log R\right) / \dot{M}_{a}$ by a factor of $\sim 7$. Reading figures from many existing wind simulations with different setups, it appears that the steady-state poloidal field configurations tend to diverge well before reaching $R \sim 3 R_{0}$ (e.g., Krasnopolsky et al. 1999; Zanni et al. 2007; Murphy et al. 2010; Stepanovs \& Fendt 2014). In reality, the situation must depend on the radial distribution of magnetic flux. For instance, if magnetic flux were strongly concentrated towards the inner disk, then one would expect a $B_{p} \propto R^{-2}$ scaling right from the wind launching region, as can be seen in Krasnopolsky et al. (2003). The X-wind model (Shu et al. 1994) constitutes

\footnotetext{
${ }^{10}$ In the unphysical situation of $q \rightarrow 0$ (hence $B_{p} \propto 1 / R$ ), no wind solution can be found.
}

an extreme case. The relatively large ejection efficiency $\xi$ in these models is likely (partially) compensated by a relatively narrow radial range in which the wind is launched, thus limiting the total wind mass loss rate. On the other hand, a quasi-self-similar field configuration (i.e., small $q$ ) would yield much smaller $\xi$, but with a much more extended radial range. Thus, the contrast in total wind mass loss rate between large and small $q$ cases may not be as dramatic as the difference in $\xi$.

\subsection{Scaling Relations}

In $\S 2.4$, we provided several scaling relations among various characteristic wind quantities that are expected in the Weber \& Davis wind $\left(\theta=0, q=\infty, c_{\mathrm{s}, \mathrm{w}} \rightarrow 0\right)$. Our models being generalizations of WD's, we test in this subsection to what extent they follow the same scalings.

In Figure 7, we test the scaling relations (24) and (26), namely, the dependence of $R_{A}$ and $\left|B_{\phi} / B_{p}\right| R_{A}$ on the mass loading parameter $\mu$ [eq. (18)]. These two relations state that large mass loading is accompanied by small $R_{A}$, and more toroidally dominated field configuration, which means that the wind is more efficient at removing 

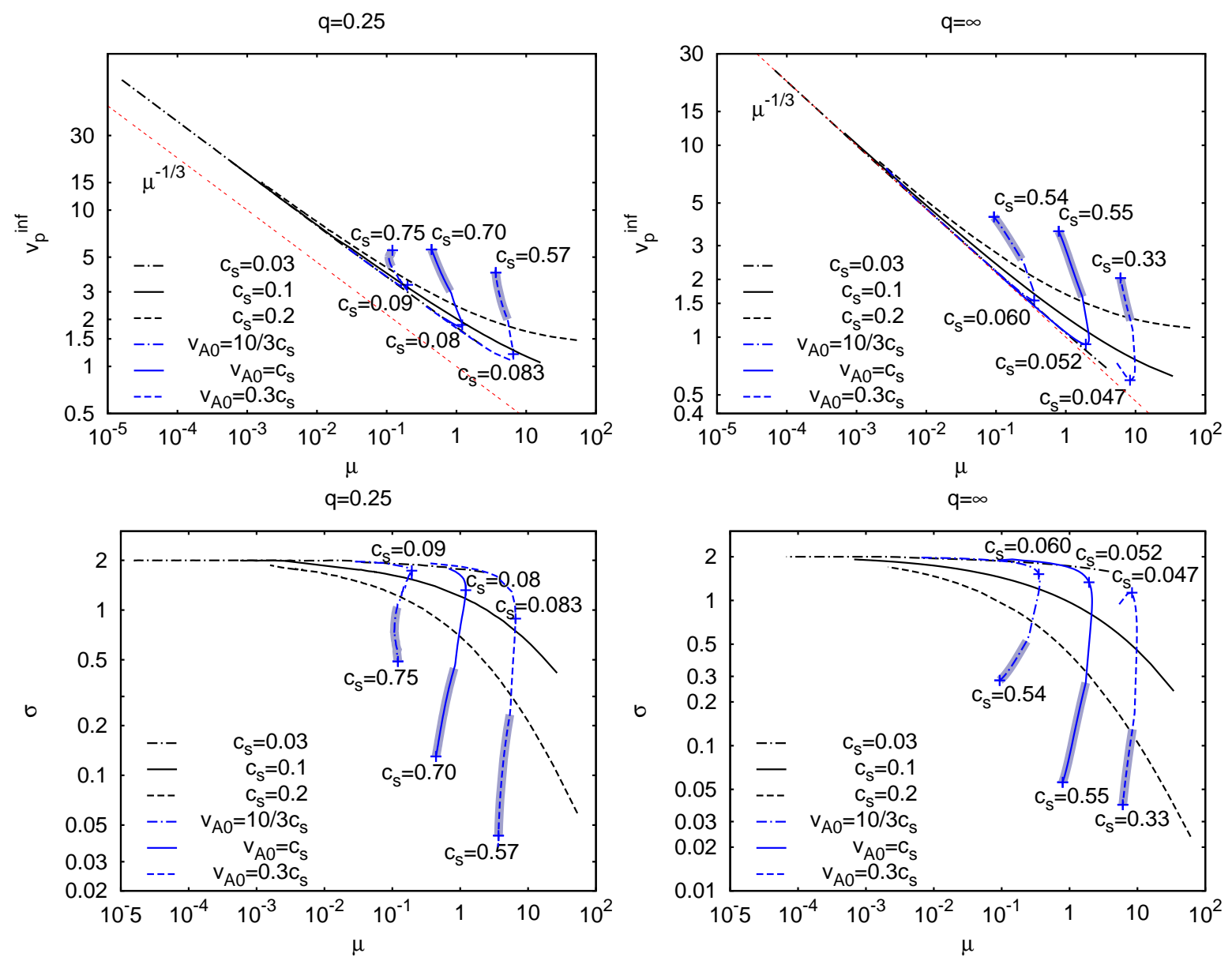

FIG. 8.- Scaling relations between pairs of wind diagnostics. The top two panels show the scaling relation between the mass loading parameter $\mu$ and the asymptotic poloidal velocity $v_{p}^{\text {inf }}$, while the bottom two panels show the relation between $\mu$ and $\sigma$ (asymptotic ratio of Poynting to kinetic energy flux). Left (right) panels correspond to wind solutions with $q=0.25(q=\infty)$. In each panel, we either choose fixed $c_{s}$, or choose $c_{\mathrm{s}, \mathrm{w}}$ to be proportional to $v_{A 0}$, as marked in the legends. The red dash-dotted line in the top panels indicates the expected scaling relation for the cold Weber \& Davis wind model (25). To guide the eye, we further label the value of $c_{\mathrm{s}, \mathrm{w}}$ at various turning and tip points. Shaded regions have solutions with $R_{s}<R_{0}$, which would violate our assumptions, but are still shown for reference.

mass than angular momentum.

We see that for the $q=\infty$ cases, the scaling relation (24) is matched almost perfectly as long as the wind is sufficiently cold $c_{\mathrm{s}, \mathrm{w}} \lesssim 0.08 v_{K}$. This relation seems to be universal regardless of field inclination. It is interesting to note that even when $v_{A 0}<c_{\mathrm{S}, \mathrm{w}}$, the relation still holds as long as $c_{\mathrm{s}, \mathrm{w}} \lesssim 0.08 v_{K}$. The relation has also been approximately recovered in global wind simulations (Zanni et al. 2007). For hotter winds with $c_{\mathrm{s}, \mathrm{w}} \gtrsim 0.08 v_{K}$, $R_{A}$ falls below what is expected from the relation at a given $\mu$. In particular, the minimum value of $R_{A}$ is 1.5 for a cold wind according to eq. (24), but this is no longer a lower limit if the wind is warm or hot.

In the case that $q=0.25$, one sees that while for a cold wind with $c_{\mathrm{S}, \mathrm{w}} \lesssim 0.08 v_{K}$, all data points line up into an almost perfect scaling relation, it is clearly offset from eq. (24). As can be partly traced from Figure 6, for fixed $v_{A 0}$ and $c_{\mathrm{s}, \mathrm{w}}$ the $q=0.25$ case gives both larger $\mu \propto \rho v_{p}$ and larger $R_{A}$ compared to the $q=\infty$ case, thus shifting the original relation towards the upper right, as is observed here. Other aspects of the relation are very similar to the $q=\infty$ case.
In the bottom panels of Figure 7 , one sees that when $q=\infty$, the asymptotic relation (26) is accurately satisfied in the strong-field limit (giving $\mu \ll 1$ ), with $\left|B_{\phi} / B_{p}\right|_{A} \rightarrow 1.5$. The weak field limit of the asymptotic relation depends on $c_{\mathrm{S}, \mathrm{w}}$, and is only achieved with $c_{\mathrm{s}, \mathrm{w}} \rightarrow 0$. In the case of $q=0.25$, there is also a welldefined relation for $\left|B_{\phi} / B_{p}\right|_{A}$ in the strong field limit, although it does not appear to approach a constant value. Similarly, the weak field limit of the relation depends on wind temperature. One general trend is that higher wind temperatures lead to smaller $\left|B_{\phi} / B_{p}\right|_{A}$. The main reason is that higher temperature provides stronger pressure support that induces sub-Keplerian rotation, which would reduce the development of toroidal fields.

In Figure 8, we further test the scaling relation (25) in the top panels. We see that the $\mu^{-1 / 3}$ scaling for terminal velocity $v_{p}^{\text {inf }}$ is very well satisfied when $q=\infty$ if the wind is cold. Enlarging the temperature at fixed $v_{A 0}$ yields larger $v_{p}^{\text {inf }}$. When $q=0.25$, our fiducial choice, the $\mu^{1 / 3}$ scaling is still very well satisfied, except for being shifted up by a factor of $\lesssim 2$, consistent with Figure 6 .

The asymptotic ratio of Poynting flux to kinetic en- 

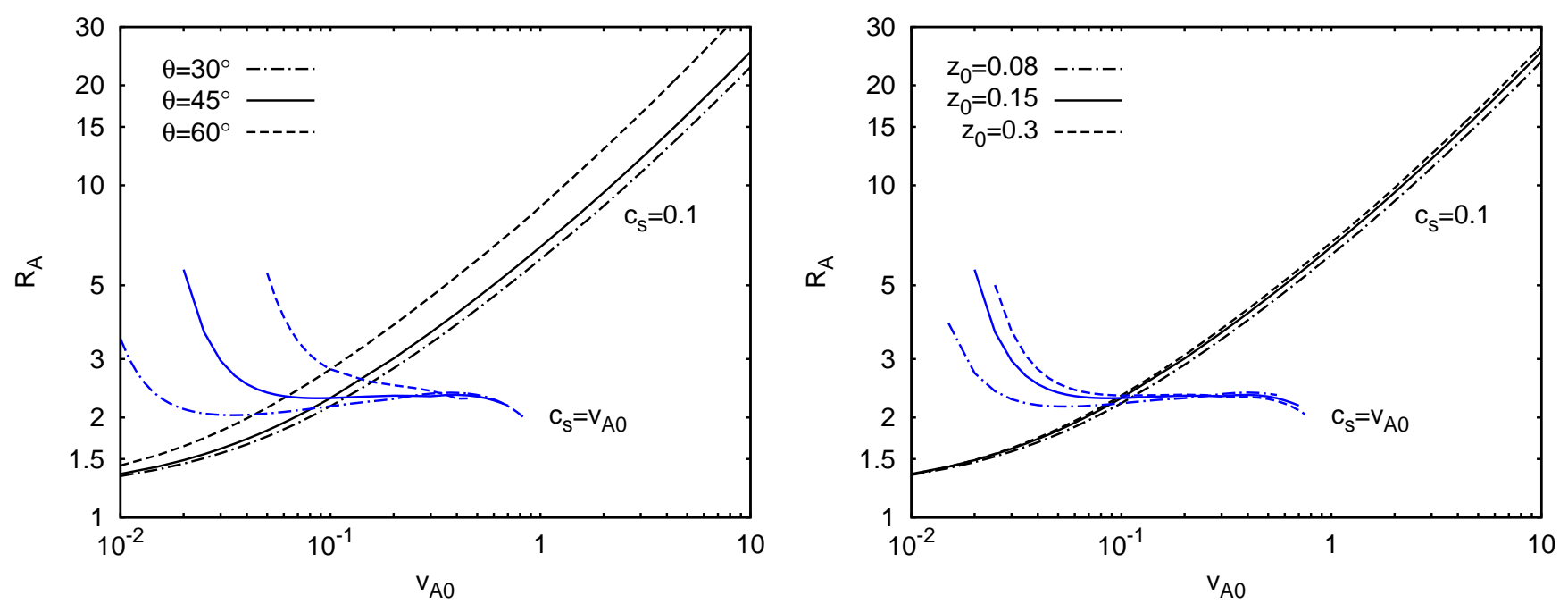

Fig. 9.- Left: Alfvén radius $R_{A}$ as a function of $v_{A 0}$ for different choices of field inclination angle $\theta$, as marked in the legend. Black lines have fixed $c_{\mathrm{S}, \mathrm{w}}=0.1$, while blue lines have fixed $c_{\mathrm{s}, \mathrm{w}}=v_{A 0}$. Other parameters are taken as fiducial (e.g., $\left.q=0.25, z_{0}=0.15\right)$. Right: same as left, but for different choices of wind base location $z_{0}$. Other parameters are taken as fiducial $\left(\right.$ e.g., $\left.q=0.25, \theta=45^{\circ}\right)$.

ergy $(\sigma)$ for various wind parameters is shown in the bottom panel of Figure 8. Regardless of the $q$ value, $\sigma$ always reaches the maximum value of 2 as long as the wind is strong (giving small $\mu$ ), or cold (small $c_{\mathrm{s}, \mathrm{w}}$ and $\left.v_{A 0}<c_{\mathrm{s}, \mathrm{w}}\right)$. Modestly warm winds with $c_{\mathrm{s}, \mathrm{w}} \gtrsim 0.1 v_{K}$ and solutions with $v_{A 0}<c_{\mathrm{s}, \mathrm{w}}$ all yield smaller Poynting flux, and the wind power is then largely kinetic.

\section{PARAMETER DEPENDENCE}

In this section, we explore the secondary parameters, whose effect on wind properties turns out usually to be minor, justifying in part the simplicity of our models.

\subsection{Dependence on Field Geometry}

We first explore the effect of the field inclination angle $\theta$ and wind-base height $z_{0}$. Since we are mostly interested in the efficiency of angular momentum transport and mass loss, we only show the dependence of $R_{A}$ on $v_{A 0}$ for various values of $\theta$ and $z_{0}$, illustrated in Figure 9 .

The inclination angle influences the effective potential that the wind flow experiences. Larger angles require the wind flow to climb out of a deeper gravitational potential well. As a result, hydrostatic support extends farther and the density at the slow critical point drops, leading to a more lightly loaded wind and more effective acceleration. This trend is clearly observed in Figure 9. Increasing (decreasing) $\theta$ always leads to larger (smaller) $R_{A}$ at fixed wind temperature. The change in $R_{A}$ is more pronounced when varying $\theta$ from $45^{\circ}$ to $60^{\circ}$ than from $30^{\circ}$ to $45^{\circ}$.

When fixing $c_{\mathrm{S}, \mathrm{w}}=v_{A 0}$, we see that the effect of different inclination angle is more significant towards lower temperature. This is simply because higher temperature helps overcome the steeper gravitational potential caused by larger $\theta$, and in general reduces the importance of gravity. For our fiducial choice $v_{A 0}=c_{\mathrm{s}, \mathrm{w}}=0.1 v_{K}$, appropriate for $R \sim 1 \mathrm{AU}$ in PPDs, the difference in $R_{A}$ among different choices of $\theta$ is modest unless the inclination angle is larger than $60^{\circ}$. Furthermore, at larger radii in PPDs, we expect $c_{\mathrm{s}, \mathrm{w}} / v_{K}$ to increase, thus making the inclination effect only a minor consideration.

The right panel of Figure 9 shows the corresponding plot for varying $z_{0}$. Clearly, the geometric location of the wind base has very little impact on the wind properties, apart from a very weak tendency to be more lightly loaded with increasing $z_{0}$. Note that here we simply vary the geometric location of the wind base $z_{0} / R_{0}$, but we do not vary $v_{A 0}$ according to (A4). The latter effect has already been incorporated in the discussions in Section 3.2. Evidently, the UV penetration depth affects the wind properties mainly by controlling the Alfvén speed $v_{A 0}$ rather than the geometric height $z_{0}$.

In $\mathrm{BP}$ and subsequent literature, it is often stated that $60^{\circ}$ is the critical angle for launching a magnetocentrifugal wind. However, this statement applies to cold winds launched from razor-thin disks $\left(c_{\mathrm{s}, \mathrm{w}}=0\right.$ and $\left.z_{0}=0\right)$. For winds of finite temperature launched from finite heights, there is no sharp inclination threshold.

One main limitation of this work is that we have prescribed the poloidal field geometry rather than solving for it self-consistently from cross-field force balance. The main effect that we miss is wind collimation, which is a natural consequence of disk wind from magnetic hoop stress, and is present in almost all self-similar wind solutions and wind simulations. The fact that our wind solutions are not very sensitive to variations in $\theta$ within $30^{\circ}-60^{\circ}$ already suggests that our main results are not sensitive to field geometry. In addition, significant collimation is typically achieved well beyond the Alfvén point, where the wind flow has largely escaped from the gravitational potential. Thus we expect essential wind properties (viz. mass loading and $R_{A} / R_{0}$ ) not to be strongly affected by the field geometry at large distances.

\subsection{Dependence on Magnetic Angular Velocity}

Heretofore we have fixed $\omega=\Omega_{K}$, which is expected to hold approximately but not strictly. A source of deviation of $\omega$ from $\Omega_{K}$ is intrinsic to the disk rotation profile in PPDs, which are externally irradiated and flared. 


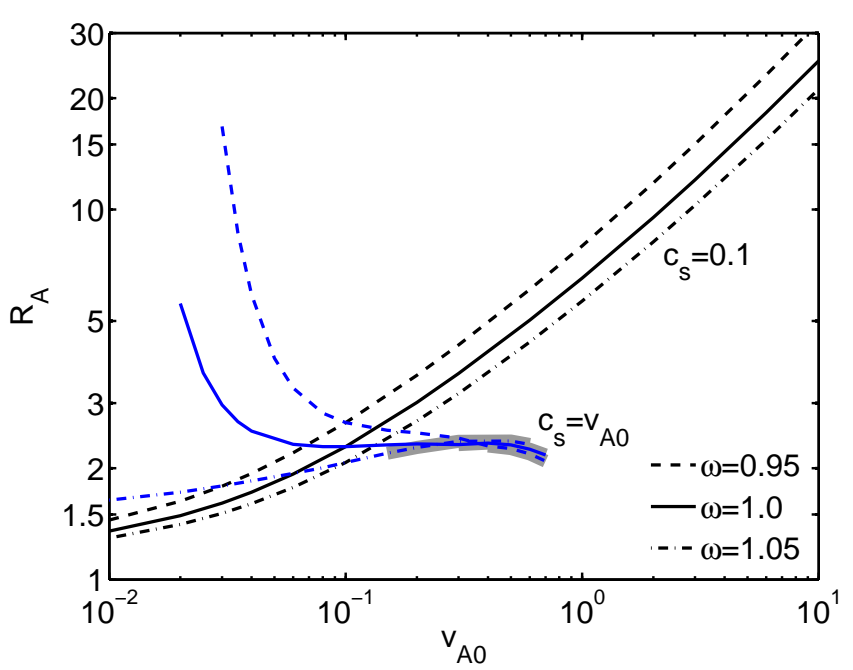

Fig. 10.- Alfvén radius $R_{A}$ versus Alfvén speed at wind base $v_{A 0}$ for different field-line angular velocities $\omega$, as marked in the legend. Black lines have fixed $c_{\mathrm{s}, \mathrm{w}}=0.1$, blue lines $c_{\mathrm{S}, \mathrm{w}}=v_{A 0}$. Other parameters are fiducial (e.g., $q=0.25, \theta=45^{\circ}$ ). Shaded regions have $R_{s}<R_{0}$, which violates our assumptions.

The midplane rotates at sub-Keplerian speed due to partial radial pressure support. On the other hand, superKeplerian rotation may occur at the disk surface where the radial pressure gradient reverses. For the standard vertically isothermal MMSN model (see Appendix A for adopted parameters), the non-Keplerian deviation is

$$
\frac{\Delta v_{\phi}}{v_{K}} \approx 1.1 \times 10^{-3}\left(\frac{13}{8} R_{\mathrm{AU}}^{1 / 2}-\frac{5}{8} \frac{z^{2}}{H^{2}} R_{\mathrm{AU}}^{-1 / 2}\right),
$$

where $R_{\mathrm{AU}}$ is radius measured in AU. For our fiducial choice of $R=1 \mathrm{AU}$ and $z_{0}=4.5 H$, we get superKeplerian rotation by about $1.2 \%$. At larger radii, we find sub-Keplerian rotation $<2 \%$ out to $\sim 120 \mathrm{AU}$.

Given these estimates, we show in Figure 10 the Alfvén radius as a function of $v_{A 0}$ for $\omega$ varying at an exaggerated $5 \%$ level (all other parameters being fiducial). We see that at fixed $c_{\mathrm{s}, \mathrm{w}}=0.1$, varying $\omega$ at this level has relatively small impact on the wind properties. Thus we do not expect wind properties to be sensitive to $\omega$.

One sees that smaller $\omega$ leads to larger $R_{A}$ (except for hotter winds as marked in shaded areas where $R_{s}<R_{0}$ and our solutions are less trustworthy). This is because smaller $\omega$ is associated with less free energy available for wind launching, requiring a larger density drop in the hydrostatic region near the wind base, and leading to a more lightly loaded wind. If sub-Keplerian rotation is substantial, wind launching can be suppressed without thermal pressure support (Shu et al. 2008). In Figure 10 , the blue lines diverge towards smaller $c_{\mathrm{s}, \mathrm{w}}$ (chosen equal to $v_{A 0}$ ), while tending to converge towards large $c_{\mathrm{s}, \mathrm{w}}$. This reflects the importance of thermal effects: colder winds are strongly affected by the rotation profile at the wind base due to energetic constraints, while warmer winds are exempt from such sensitive dependence. Therefore, external heating of PPD surface eases the wind launching process and makes it suffer much less from deviations from Keplerian rotation.

\subsection{Dependence on Equation of State}

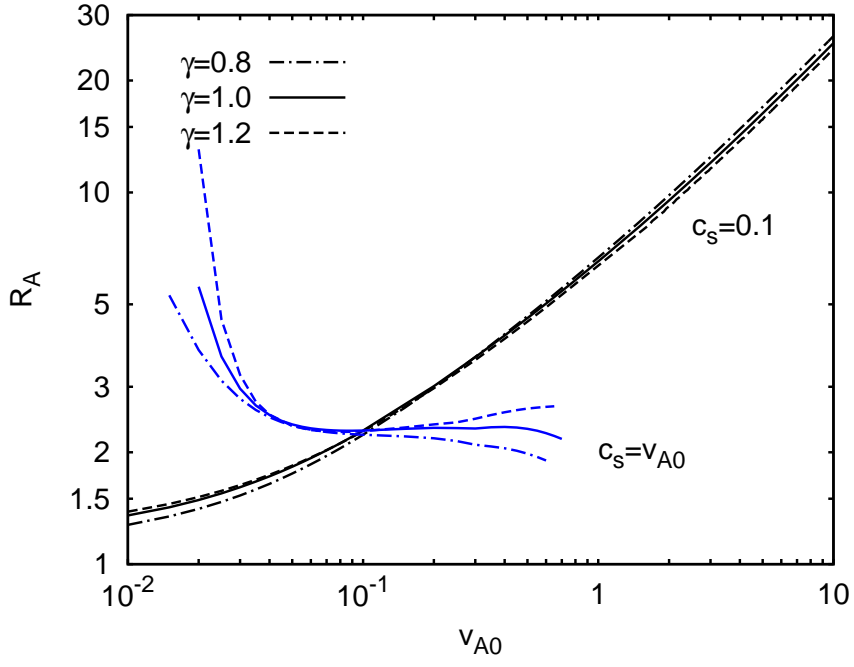

FIG. 11.- Like Fig. 10 but for differing polytropic indices $\gamma$ rather than angular velocities.

Our discussion of the role of thermodynamics has been restricted to an isothermal equation of state. To justify this choice, here we substitute a polytropic equation of state with $P=K \rho^{\gamma}$. This change enters the Bernoulli equation through the enthalpy term, which now becomes

$$
h=\frac{\gamma}{\gamma-1} K \rho^{\gamma-1} .
$$

We set the parameter $K$ such that $P / \rho=c_{\mathrm{s}, \mathrm{w}}^{2}$ at the wind base. We consider two adiabatic indices: $\gamma=0.8$ and 1.2. The former mimics the increase of gas temperature above the wind base as a result of additional heating sources that penetrate no less deeply than FUV (e.g., extreme UV). The latter $(\gamma=1.2)$ leans toward the opposite case where adiabatic cooling reduces gas temperature along wind field lines.

Figure 11 illustrates the results of these different equations of state. Evidently the Alfvén radius is very insensitive to variations in $\gamma$. We have also checked the mass loading parameter $\mu$ and $\left|B_{\phi} / B_{p}\right|$ at the wind base, and confirm that they are all very similar for different choices of $\gamma \mathrm{s}$. Looking at Figure 2, the density drop from the wind base (near the slow point) to the Alfvén point in our fiducial solution amounts to more than 2 orders of magnitude. Such density drop leads to temperature variations for our different choices of $\gamma \mathrm{s}$ by a factor more than 6 , yet major wind properties remain similar to the isothermal case.

Different choices of $\gamma$ do affect a few other wind properties. In particular, for the somewhat unphysical choice $\gamma=0.8$, the gas temperature increases with decreasing density. As a result, the gas never reaches a terminal speed but keeps accelerating on its own: magnetic field is not required for wind launching, a situation analogous to pure photo-evaporation. The $\gamma=1.2$ case, on the other hand, shows very little difference in terminal velocity from the isothermal one. Also, the location of fast magnetosonic point is extremely sensitive to $\gamma$ : $R_{f}=64.2,554.5$ and $7.70 \times 10^{4}$ for $\gamma=0.8,1$ and 1.2 , respectively. Despite such differences, we have already seen that our most interesting quantity, the Alfvén ra- 
dius, and the associated wind-driven accretion rate and wind mass loss rate, are very insensitive to thermodynamics at $R \gg R_{0}$.

\section{DISCUSSION}

\subsection{Observations of PPD Outflows}

Disk winds from PPDs likely correspond to the lowvelocity component of outflow (as opposed to the highvelocity jet), whose signatures have now been routinely inferred from blue-shifted emission line profiles such as from CO, OI and NeII lines (e.g., Hartigan et al. 1995; Pascucci \& Sterzik 2009; Pontoppidan et al. 2011; Bast et al. 2011; Herczeg et al. 2011; Sacco et al. 2012; Rigliaco et al. 2013; Brown et al. 2013; Natta et al. 2014). The blueshift is typically a few $\mathrm{km} \mathrm{s}^{-1}$, comparable to the disk sound speed at sub-AU to AU scales, and is consistent with MHD wind flows near the launching region.

One crucial parameter in the wind-driven accretion framework is the ratio of wind mass loss rate $\dot{M}_{\text {wind }}$ to the wind-driven accretion rate $\dot{M}_{\text {acc }}$. A correlation between the two quantities was identified in early observational studies (Cabrit et al. 1990; Hartigan et al. 1995). Following later calibration of accretion rates (Gullbring et al. 1998; Muzerolle et al. 1998), a canonical number of $\dot{M}_{\text {wind }} / \dot{M}_{\text {acc }} \approx 0.1$ has been established, i.e., mass loss rate is a small fraction of accretion rate. However, the mass-outflow rate obtained by Hartigan et al. (1995) corresponds to the high-velocity outflow, which most likely originates from a jet launched close to the protostar. While low-velocity disk winds were ubiquitously detected in Hartigan et al. (1995), reliable estimates of the mass-flow rate were not feasible. Recently, Natta et al. (2014) modeled the low-velocity component of the blueshifted forbidden lines from a sample of T-Tauri stars, and estimated $\dot{M}_{\text {wind }} / \dot{M}_{\text {acc }} \approx$ $0.1-1$. Estimates of mass outflow rates from more embedded protostars by Watson et al. (2015) also suggest the ratio of $\dot{M}_{\text {wind }} / \dot{M}_{\text {acc }}$ in the similar range. Studies by Klaassen et al. (2013) and Salyk et al. (2014) for two individual sources (HD 163296 and AS 205N) using the Atacama Large Millimeter Array (ALMA) both indicate significant mass outflow and again suggest $\dot{M}_{\text {wind }} / \dot{M}_{\text {acc }} \approx 0.1-1$ (with large uncertainties).

Overall, current observational constraints, although limited and bearing large uncertainties, agree with our suggested scenario that PPDs lose mass at a considerable fraction of the accretion rate. In the future, it will be crucial to combine observational and modeling efforts to reduce the uncertainties and better constrain the physical nature of PPD disk winds.

\subsection{Magneto-photoevaporation?}

As mentioned in the introduction, mass loss from PPDs has conventionally been attributed to photoevaporation. This is a purely hydrodynamic process whereby the disk surface is heated by external radiation to sufficiently high temperatures to launch a thermal wind. Early models of photoevaporation considered heating from extreme UV (EUV, Hollenbach et al. 1994; Font et al. 2004; Alexander et al. 2006a). These calculations gave mass-loss rates of the order of $10^{-10}-10^{-9} M_{\odot} \mathrm{yr}^{-1}$, and when coupled with a viscously evolving disk, can lead to rapid disk dispersal (Clarke et al. 2001; Alexander et al. 2006b). Later theoretical calculations included heating from X-rays (Alexander et al. 2004; Ercolano et al. 2008, 2009; Owen et al. 2010) and far-UV (Adams et al. 2004; Gorti \& Hollenbach 2009; Gorti et al. 2009, 2015) with increasing levels of complexity. These works demonstrated that due to deeper penetration, X-ray and FUV can drive stronger winds and mass-loss rates $\sim 10^{-9}-$ $10^{-8} M_{\odot} \mathrm{yr}^{-1}$, which approach typical disk accretion rates.

There are two major differences between the photoevaporation and MHD-disk-wind scenarios. First, a thermal wind exerts no torque on the disk, and hence the mass-loss rate is unrelated to the accretion process. Second, magnetic forces plays a dominant role in wind driving in an MHD wind (see Figure 4), and with the additional push, likely yield larger mass loss rate. Unfortunately, it may not be easy to distinguish the two scenarios observationally. Along flow streamlines, specific angular momentum is conserved for a purely thermal wind. In MHD winds, unless the poloidal field is strong and the wind lightly loaded, specific angular momentum is also approximately conserved, as seen in Fig. 3.

In reality, it is probably meaningless to argue for which scenario is more correct. It is clear that both external irradiation and magnetic fields play indispensable roles in the wind launching and acceleration processes. In this regard, we feel that "magneto-thermal wind" or "magnetophotoevaporation" may be more appropriate names to describe the mass loss process from PPDs.

\subsection{Global Evolution of Protoplanetary Disks}

Our simple 1D wind model represents a starting point towards constructing a global model of PPD evolution that predicts the rate of angular momentum transport and mass loss at all disk radii.

We first note that while our model is mainly motivated from studies of PPD gas dynamics in the inner disk, our wind framework is likely equally applicable to the outer disk as well ( $R \gtrsim 10$ AU). At the outer disk, ambipolar diffusion is the sole dominant non-ideal MHD effect, which substantially reduces the coupling between gas and magnetic fields in the disk interior, and damps or suppresses the MRI (Bai \& Stone 2011; Simon et al. 2013b,a). Efficient angular momentum transport requires the presence of net vertical magnetic flux, which in the mean time launches a disk wind. Far UV ionization again has been found to be crucial in these processes, and if penetrating sufficiently deeply, can lead to vigorous MRI turbulence at disk surface (Simon et al. 2013a; Bai 2015). On the other hand, non-detection of surface turbulence around the disk HD 163296 by Flaherty et al. (2015) may suggest less deep FUV penetration, leading to a largely laminar disk upon which our wind framework can be readily applied.

To facilitate an effort towards constructing a global model of wind-driven PPD evolution, we show in Figure 12 the dependence of wind-driven accretion rate $\dot{M}_{\text {acc }}$, and wind mass loss rate per logarithmic radius $d \dot{M}_{\text {wind }} / d \ln R$, as a function of the main wind parameters, based on Fig. 5 and eqs. (19) \& (21). The accretion/outflow rates are in dimensionless units and are 

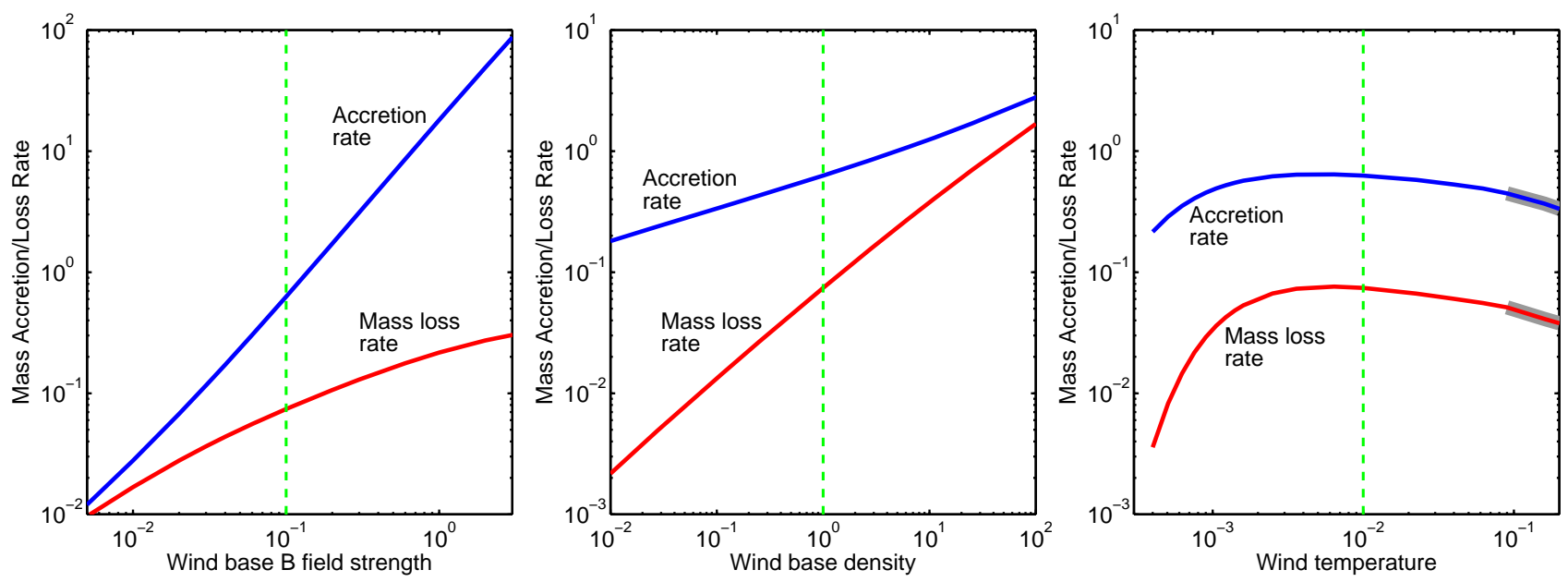

FIG. 12.- Schematic dependence of wind-driven accretion rate and mass-loss rate on the main physical parameters of magneto-thermal winds, constructed based on fiducial wind model parameters shown in Fig. 5 (choosing $c_{\mathrm{s}, \mathrm{w}}=0.1 v_{K}$ for left and middle panels and $c_{\mathrm{S}, \mathrm{w}}=v_{A 0}$ for the right panel) and eqs. (19) and (21). Our standard wind solution $\left(v_{A 0}=c_{\mathrm{S}, \mathrm{w}}=0.1 v_{K}\right)$ is marked by green dashed lines, corresponding to an accretion rate $\sim 10^{-8} M_{\odot} \mathrm{yr}^{-1}$ at $1 \mathrm{AU}$. The main parameters are poloidal magnetic field strength at the wind base $B_{p 0}$ (left, in units of $v_{A 0}$ ), density of the wind base $\rho_{b}$ (middle, reflecting the penetration depth of FUV ionization, in arbitrary units), and wind temperature $T_{w}$ (right, in units of $\left.c_{\mathrm{s}, \mathrm{w}}^{2} / v_{K}^{2}\right)$.

freely scalable. A vertical green dashed line in each panel marks our standard parameters, corresponding to $R=1 \mathrm{AU}$ with fiducial field strength and FUVpenetration depth, and resulting in a wind-driven accretion rate $\sim 10^{-8} M_{\odot} \mathrm{yr}^{-1}$. In the left and middle panels, we break the the dependence of accretion/massloss rate on $v_{A 0}$ into the dependence on poloidal field strength $B_{p 0}$ and wind-base density $\rho_{b}$ separately. The right panel varies the wind temperature $\left(\propto c_{\mathrm{s}, \mathrm{w}}^{2}\right)$. The middle panel aims to illustrate the role of FUV penetration depth, where deeper penetration leads to larger wind-base density.

We see that increasing poloidal field strength increases both accretion and mass loss rates, with the former increasing much faster, corresponding to larger Alfvén radius. Fixing the field strength while enhancing FUV penetration depth (enhanced $\rho_{b}$ ) also leads to both higher accretion and mass-loss rates. In this case, the system develops a more heavily loaded wind, and winds up toroidal field more (i.e., larger $B_{\phi}$ ), but it is the mass-loss rate that increases faster. Variations in wind temperature, on the other hand, appear to only weakly affect the wind properties. This is mainly because the wind base is in pressure balance with the disk interior. For a steadystate wind, an increase in temperature is compensated by lower density, which does not alter the relative importance between gas and magnetic pressure (or $v_{A 0} / c_{\mathrm{s}, \mathrm{w}}$ ).

Acknowledging all the uncertainties discussed in $\S 2.1$ for our local wind framework, we expect such an approach to be able to capture the physical essence of winddriven evolution of PPDs. We note that a recent effort by Armitage et al. (2013) on PPD evolution adopted wind torques based on scalings from local disk simulations with turbulence, and neglected disk mass loss. Our wind model have incorporated the physics at global scales, and the thorough exploration of parameter space allows us to better account for the controlling effects of disk evolution. In the mean time, the schematic plots in Figure
12 highlight the importance of better understanding two issues.

The first is the transport of magnetic flux in PPDs, which determines the radial profile of poloidal field strength and its evolution. So far, most studies conform to the framework of inward advection by accretion and outward transport by turbulent diffusion assuming magnetic field embedded in vacuum beyond the disk (e.g., Lubow et al. 1994a; Okuzumi et al. 2014; Guilet \& Ogilvie 2014). The presence of wind plasma with wind-driven accretion may significantly change the picture (Cao \& Spruit 2013). In addition, non-ideal MHD effects within the disk, especially the polaritydependent Hall effect, may also play an important role in magnetic flux transport, as already hinted by local studies (Bai 2014). On the other hand, regardless of the details of magnetic-flux transport, the fact that accretion rates of young stellar objects (YSOs) decrease with age (Fedele et al. 2010; Sicilia-Aguilar et al. 2010) implies that the disk must lose magnetic flux over time, which may permit phenomenological considerations.

The second issue is UV radiative transfer, the associated photochemistry, and their coupling to wind dynamics, which largely determine the location of the wind base. Despite increasing sophistication, most photochemical modeling efforts so far have assumed hydrostatic equilibrium (e.g., Nomura \& Millar 2005; Woitke et al. 2009; Bethell \& Bergin 2011; Walsh et al. 2012), and this is also the case for FUV photoevaporation studies (e.g., Gorti et al. 2009). Density profiles in the MHD wind-launching region deviate from being purely hydrostatic due to substantial magnetic pressure support, and asymptote to a $\rho \propto R^{-2}$ profile in the wind, which differs substantially from Gaussian-like hydrostatic profiles. Consider a situation with significant wind mass loss (say $\sim 10^{-8} M_{\odot} \mathrm{yr}^{-1}$ ) with characteristic velocity of $\sim 5 \mathrm{~km} \mathrm{~s}^{-1}$ (low velocity component) at a characteristic radius of $\sim 1$ AU: the wind column 
density would reach $\sim 0.015 \mathrm{~g} \mathrm{~cm}^{-2}$, comparable to the expected penetration depth for far-UV photons. Such shielding would make wind launching occur at larger vertical height, thus reducing mass loss, especially towards larger radii. This shielding effect may also directly affect observational diagnostics of winds, as has been discussed with regard to the survival of molecules in disk winds (Panoglou et al. 2012).

Additional complexities arise from stability considerations. Wind stability has been discussed in §3.1.1. Another stability issue concerns the disk itself when accretion is wind-driven (Lubow et al. 1994b; Cao \& Spruit 2002; Königl 2004; Moll 2012; Lesur et al. 2013). Claims for both stability and instability exist, although these studies have simplified the disk physics relevant to PPDs, and the situation is far from being clear. Both wind and disk stability issues call for realistic 3D simulations.

\subsection{Implications for Planet Formation}

Disk winds may affect many aspects of planet formation by influencing the global evolution of PPDs through angular-momentum transport and mass loss. The fact that we expect PPDs to lose mass quickly has a more direct consequence on planet formation: it will promote planetesimal formation.

Planetesimal formation remains the least understood process in the theory of planet formation (see review by Chiang \& Youdin 2010). Among possible mechanisms, streaming instability (Goodman \& Pindor 2000; Youdin \& Goodman 2005) appears to be the most promising. However, to form planetesimals via the streaming instability, the dust-to-gas ratio must be enhanced by a factor of several above solar abundance, $\sim$ 0.01 (Johansen et al. 2007, 2009; Bai \& Stone 2010a,b). This requirement poses a strong challenge for planetesimal formation in the terrestrial-planet-forming region within the snow line (Drążkowska \& Dullemond 2014).

In the presence of a disk wind, gas is removed from several scale heights above disk midplane. With the disk being largely laminar or weakly turbulent, most dust grains are expected to settle toward the disk midplane. Therefore, we expect the wind to primarily remove gas with depleted dust content, effectively enhancing the dust-to-gas mass ratio. Gorti et al. (2015) explored this idea within the framework of photoevaporation and found that substantial enhancement dust-to-gas ratios can be achieved. We expect similar conclusions to hold in the MHD-wind framework, and this effect may be even more prominent given the further enhanced mass-loss rate. Therefore, planetesimal formation via the streaming instability may become feasible in the inner region of PPDs.

Using CO as a gas tracer, Williams \& Best (2014) reported evidence of enhanced dust-to-gas ratios for a group of PPDs. Their results mainly apply to the outer parts of disks, which contain the bulk of the mass reservoir. Although the reliability of their gas-mass estimates remains questionable, the estimates suggest that wind mass loss may also be significant in the outer disk.

\subsection{Connection to Black Hole Accretion Disks}

While we have focused on magnetized disk winds from PPDs, the results presented here may also shed light on accretion disks surrounding compact objects, espe- cially black holes (BHs). While $\mathrm{BH}$ accretion disks are fully MRI turbulent, local MHD simulations show that wind launching in the presence of net vertical magnetic flux typically occurs at several scale heights above the disk midplane (Suzuki \& Inutsuka 2009; Fromang et al. 2013). Global simulations of hot accretion flows have also identified magnetic forces for driving disk outflows (Yuan et al. 2012, 2015). Moreover, magnetic fields become more ordered as net vertical field becomes stronger (Bai \& Stone 2013a), permitting a quasi-steady-state model construction.

In the context of active galactic nuclei (AGN) disks hosting supermassive BHs, powerful winds are commonly observed in broad absorption lines, with inferred mass loss rates comparable to or even higher than accretion rates (see Crenshaw et al. 2003 for a review). While radiation force likely plays a dominant role in many cases for wind driving, not all AGN winds can be explained by radiation driving alone (see Proga 2007 for a review), and magnetic driving also appears to be essential (Konigl \& Kartje 1994; de Kool \& Begelman 1995; Everett 2005; Kraemer et al. 2005). In the context of Xray binaries hosting stellar mass black holes, disk wind signatures are commonly observed in the disk-dominated soft states (e.g., Neilsen \& Lee 2009; King et al. 2012; Miller et al. 2012; Ponti et al. 2012), again with mass loss rate comparable to accretion rate. While very high degree of ionization requires strong X-ray illumination, photoionization modeling shows that neither radiation nor thermal pressure can account for the wind kinematics, hence magnetic driving is inevitable (e.g., Miller et al. 2006; Neilsen \& Homan 2012). In both contexts, provided the estimates for the size of the wind launching region, wind temperature, together with wind velocity and mass flux based on photoionization modeling, it is possible to place useful constraints on disk magnetization from our wind framework. This parameter is of great theoretical interests, and likely plays a decisive role in overall disk evolution.

\section{SUMMARY AND CONCLUSIONS}

In this paper, we have constructed a simple 1D model for the kinematics of magnetized winds launched from PPDs. It is motivated by local simulations of PPD gas dynamics which included detailed microphysics, as well as theoretical and observational studies of PPD photoevaporation. We aim to unite the two scenarios of PPD mass loss into a joint theoretical framework, which we coin magneto-photoevaporation or magneto-thermal winds, since both magnetic and thermal effect play a major role in wind launching and kinematics. Key ingredients in this model are the following:

- Wind launching takes place in the warm disk surface that is well ionized by (far-) UV radiation, while the cold and poorly ionized disk interior does not participate (see Figure 1).

- The geometry and strength of poloidal magnetic field lines are prescribed, as is thermodynamics (sound speed); these serve as main parameters.

- The model is built upon conservation laws in axisymmetric steady state and ideal MHD, and is a 
direct generalization of the Weber \& Davis (1967) wind.

We choose our fiducial wind model to mimic wind launched from $\sim 1 \mathrm{AU}$ with a field strength that would drive an accretion rate $\sim 10^{-8} M_{\odot} \mathrm{yr}^{-1}$. But the model scalable, and we have made a thorough exploration of the parameter space surrounding the fiducial model. Most of our investigation has focused on the wind mass-loss rate and wind-driven accretion rate, whose ratio is directly connected to the Alfvén radius [see eq. (17)]. The main findings are best summarized in Figure 12, including these:

- Increasing the poloidal field strength rapidly increases the accretion rate, and less rapidly the mass-loss rate, making the wind more lightly loaded.

- Increasing the FUV penetration depth rapidly increases the mass-loss rate, and less rapidly the mass accretion rate, making the wind more heavily loaded.

- Increasing only the wind temperature modestly affect boths the accretion rate and mass loss rate.

In essence, the wind kinematics is largely determined by the ratio of poloidal Alfvén velocity $v_{A 0}$ and sound speed at the wind base $c_{\mathrm{S}, \mathrm{w}}$ (see Figure 5). Strongly magnetized winds $\left(v_{A 0}>c_{\mathrm{S}, \mathrm{w}}\right)$ have larger Alfvén radii and are lightly loaded. We also find that the classical centrifugal driving applies only when $v_{A 0} \gg c_{\mathrm{s}, \mathrm{w}}$; otherwise the wind driving mechanism is largely due to gradients in the toroidal magnetic pressure as is most likely the case for PPD winds. This fact raises concerns about the stability of PPD winds that demand further investigation.
We find that the general wind properties are not very sensitive to the geometric shape of the field lines, nor to the modest level of sub-Keplerian or super-Keplerian disk rotation. However, they do depend relatively strongly on the poloidal field strength and how rapidly $B_{p}$ achieves a $R^{-2}$ rather than $R^{-1}$ profile. This in turn may depend on how poloidal magnetic flux is distributed in the disk. We expect radially concentrated (extended) flux distributions to yield relatively smaller (larger) $R_{A}$ and greater (less) local mass loading. Yet the difference in global mass-loss rate may not be so dramatic because the more heavily loaded winds may be confined to smaller radial ranges by their more concentrated flux distributions.

In addition, we find that most kinematic scaling laws in the cold Weber \& Davis wind model can be extended to PPD disk winds, with certain renormalizations, as long as the wind is relatively cold $\left(c_{\mathrm{s}, \mathrm{w}} / v_{K} \lesssim 0.1\right)$.

Overall, our results suggest that due to the dominant role played by magnetic fields, PPDs are likely lose mass more quickly than previously thought (from pure photoevaporation), and reach a considerable fraction of the wind-driven accretion rate. Bearing in mind the uncertainties and caveats, the connection between wind-driven accretion and wind mass loss and their dependence on disk parameters that we have studied in this work may facilitate future development toward a realistic theoretical framework for global PPD structure and evolution.

We thank Uma Gorti, Geoffroy Lesur and Eve Ostriker for useful discussions. XNB acknowledges support from Institute for Theory and Computation (ITC) at Harvard-Smithsonian Center for Astrophysics. JG acknowledges support from the NASA Origins of Solar Systems program via grant NNX10AH37G. FY acknowledges support by the NSF of China (grants 11133005 and 11573051), and the Strategic Priority Research Program The Emergence of Cosmological Structures of CAS (grant XDB09000000).

\section{APPENDIX}

\section{A. ESTIMATE OF FIDUCIAL WIND PARAMETERS}

PPDs show signatures of active accretion onto the protostar, with typical accretion rate on the order of $10^{-8} M_{\odot}$ $\mathrm{yr}^{-1}$ (Hartmann et al. 1998), which requires efficient transport of disk angular momentum. As discussed in Wardle (2007) and Bai \& Goodman (2009), this fact alone places an important constraint on the magnetic field strength in PPDs. For wind-driven accretion, accretion rate is directly proportional to the magnetic torque exerted at the disk surface $\dot{M} \approx 2\left|B_{z} B_{\phi}\right| R / \Omega$, and correspondingly, the expected field strength at disk surface is

$$
B_{p 0} \approx 0.065 G \cdot \dot{M}_{-8}^{1 / 2}\left(\frac{B_{p}}{B_{\phi} \sin \theta}\right)^{1 / 2} R_{\mathrm{AU}}^{-5 / 4}
$$

for disks orbiting a solar mass star, where $\dot{M}_{-8}$ is disk accretion rate normalized to $10^{-8 \pm 1} M_{\odot} \mathrm{yr}^{-1}$, and $R_{\mathrm{AU}}$ is the cylindrical radius normalized to $1 \mathrm{AU}$. We note that transport of angular momentum vertically by disk wind is more efficient than radially by Maxwell stress by a factor of $\sim R / H$. Therefore, for a given accretion rate, the required field strength for wind-driven accretion is weaker. Recently, Fu et al. (2014) reported the magnetic field strength of the early solar nebular deciphered from the Semarkona meteorite. Within uncertainties, the inferred (midplane) field strength is relatively weak and is more compatible with the wind-driven accretion scenario.

To non-dimensionize this field strength at the wind base for our model, we apply the standard minimum-mass solar nebular (MMSN) disk model (Weidenschilling 1977; Hayashi 1981) with

$$
\Sigma_{\mathrm{MMSN}}=1700 R_{\mathrm{AU}}^{-3 / 2} \mathrm{~g} \mathrm{~cm}^{-2}, \quad T=280 R_{\mathrm{AU}}^{-1 / 2} \mathrm{~K} .
$$

Here, disk temperature is assumed to be vertically isothermal, and it refers to the "cold" region marked in Figure 1, e.g., disk interior. The normalized disk scale height is given by $H / R=c_{s} / v_{K} \approx 0.034 R_{\mathrm{AU}}^{1 / 4}$, where $c_{s}$ is the isothermal 
sound speed in the disk interior.

We first discuss the location of the wind base $z_{0}$, corresponding to the FUV ionization front. Assuming a FUV penetration of depth of $\gtrsim 0.01 \mathrm{~g} \mathrm{~cm}^{-2}$ (Perez-Becker \& Chiang 2011) and hydrostatic equilibrium, the wind base location is approximately at $z=4.4 H$ for $R_{0}=1 \mathrm{AU}$. This is further complicated by two factors. First, the gas in the wind zone is expected to be denser than from hydrostatic equilibrium, and hence the FUV ionization front should be located higher. Local isothermal wind simulations of Bai \& Stone (2013b) find $z_{0} \sim 4.6 \mathrm{H}$. Second, the gas in the wind zone is heated to become less dense, which counter-balances the previous effect to bring the FUV ionization front lower. Based on these considerations, we place $z_{0}$ at around $\sim 4.5 \mathrm{H}$ for wind launched from $R_{0}=1 \mathrm{AU}$. For wind launched from other radii, assuming constant FUV penetration depth, we expect $z_{0} / H$ be smaller toward larger radii (if shielding of FUV by the wind flow launched from smaller radii is negligible).

Hydrostatic equilibrium applies in the disk interior within $z= \pm z_{0}$. The gas density on the disk side of the wind base is

$$
\rho_{b 0} \approx 1.4 \times 10^{-9}\left(\frac{\Sigma}{\Sigma_{\mathrm{MMSN}}}\right) R_{\mathrm{AU}}^{-11 / 4} \exp \left[-\frac{1}{2}\left(\frac{z_{0}}{H}\right)^{2}\right] \mathrm{g} \mathrm{cm}^{-3} .
$$

Using Equation (A1), we obtain the plasma $\beta$ for the poloidal field at wind base

$$
\beta_{p 0}=\frac{\rho_{b 0} c_{s}^{2}}{B_{p 0}^{2} / 8 \pi}=2 \frac{c_{s}^{2}}{v_{A p}^{2}} \approx 8.2 \times 10^{4} \dot{M}_{-8}^{-1} \exp \left[-\frac{1}{2}\left(\frac{z_{0}}{H}\right)^{2}\right]\left(\frac{\Sigma}{\Sigma_{\mathrm{MMSN}}}\right)\left(\frac{B_{p}}{B_{\phi} \sin \theta}\right)^{-1} R_{\mathrm{AU}}^{-3 / 4},
$$

where $v_{A p}$ is the poloidal Alfvén velocity on the disk side of the wind base. Despite the jump of gas temperature across the wind base into the wind zone, $\beta_{p 0}$ is unaltered across the wind base, because both $B_{p}$ and gas pressure must vary smoothly. Therefore, the ratio $v_{A p} / c_{s}=v_{A 0} / c_{\mathrm{S}, \mathrm{w}}$ is unaltered across the wind base $\left(v_{A 0}, c_{\mathrm{s}, \mathrm{w}}\right.$ are the counterparts of $v_{A p}, c_{s}$ in the wind zone).

Taking $z_{0}=4.5 H$, we find $\beta_{p 0} \approx 3.3$ and hence $v_{A 0} \approx 0.8 c_{\mathrm{s}, \mathrm{w}}$ assuming all other dimensionless ratios are of order unity. This number should be taken as an order of magnitude estimate, given the super-exponential dependence on $z_{0}$ as well as uncertainties in other parameters. Therefore, we choose $v_{A 0}=c_{\mathrm{s}, \mathrm{w}}$ in our fiducial calculations, while also explore a number of variations with $v_{A 0} / c_{\mathrm{s}, \mathrm{w}} \sim 0.3-10$.

In our calculations, velocities are normalized to $v_{K}$. At the fiducial radius of $R=1 \mathrm{AU}$, gas temperature rapidly increases from $\lesssim 300 \mathrm{~K}$ in the disk interior to a few thousand K into the FUV layer (e.g., Walsh et al. 2010). Fiducially, we consider a factor of $\sim 10$ increase in gas temperature across the wind base/FUV ionization front. This corresponds to a factor $\sim 3$ increase in sound speed, giving $c_{\mathrm{s}, \mathrm{w}} \approx 3 c_{s} \approx 0.1 v_{K}$ at $1 \mathrm{AU}$. We also explore a much wider range of $c_{\mathrm{s}, \mathrm{w}}$ in our calculations.

\section{REFERENCES}

Adams, F. C., Hollenbach, D., Laughlin, G., \& Gorti, U. 2004, ApJ, 611, 360

Aikawa, Y. \& Nomura, H. 2006, ApJ, 642, 1152

Akimkin, V., Zhukovska, S., Wiebe, D., Semenov, D. Pavlyuchenkov, Y., Vasyunin, A., Birnstiel, T., \& Henning, T. 2013, ApJ, 766, 8

Alexander, R., Pascucci, I., Andrews, S., Armitage, P., \& Cieza, L. 2014, Protostars and Planets VI, 475

Alexander, R. D., Clarke, C. J., \& Pringle, J. E. 2004, MNRAS, 354,71

-. 2006a, MNRAS, 369, 216

-. 2006b, MNRAS, 369, 229

Anderson, J. M., Li, Z.-Y., Krasnopolsky, R., \& Blandford, R. D. 2005, ApJ, 630, 945

Armitage, P. J. 2011, ARA\&A, 49, 195

Armitage, P. J., Simon, J. B., \& Martin, R. G. 2013, ApJ, 778, L14

Bai, X.-N. 2011, ApJ, 739, 50

—. 2013, ApJ, 772, 96

-. 2014, ApJ, 791, 137

-. 2015, ApJ, 798, 84

Bai, X.-N. \& Goodman, J. 2009, ApJ, 701, 737

Bai, X.-N. \& Stone, J. M. 2010a, ApJ, 722, 1437

—. 2010b, ApJ, 722, L220

-. 2011, ApJ, 736, 144

—. 2013a, ApJ, 767, 30

-. 2013b, ApJ, 769, 76

Balbus, S. A. \& Hawley, J. F. 1991, ApJ, 376, 214

Bast, J. E., Brown, J. M., Herczeg, G. J., van Dishoeck, E. F., \& Pontoppidan, K. M. 2011, A\&A, 527, A119

Bergin, E. A., Aikawa, Y., Blake, G. A., \& van Dishoeck, E. F. 2007, Protostars and Planets V, 751

Bethell, T. J. \& Bergin, E. A. 2011, ApJ, 739, 78
Blandford, R. D. \& Payne, D. G. 1982, MNRAS, 199, 883

Brown, J. M., Pontoppidan, K. M., van Dishoeck, E. F., Herczeg, G. J., Blake, G. A., \& Smette, A. 2013, ApJ, 770, 94

Cabrit, S., Edwards, S., Strom, S. E., \& Strom, K. M. 1990, ApJ, 354,687

Cao, X. \& Spruit, H. C. 2002, A\&A, 385, 289

—. 2013, ApJ, 765, 149

Casse, F. \& Ferreira, J. 2000, A\&A, 361, 1178

Casse, F. \& Keppens, R. 2002, ApJ, 581, 988

—. 2004, ApJ, 601, 90

Chiang, E. \& Youdin, A. N. 2010, Annual Review of Earth and Planetary Sciences, 38, 493

Clarke, C. J., Gendrin, A., \& Sotomayor, M. 2001, MNRAS, 328, 485

Combet, C. \& Ferreira, J. 2008, A\&A, 479, 481

Contopoulos, J. \& Lovelace, R. V. E. 1994, ApJ, 429, 139

Crenshaw, D. M., Kraemer, S. B., \& George, I. M. 2003,

ARA\&A, 41, 117

de Kool, M. \& Begelman, M. C. 1995, ApJ, 455, 448

Drążkowska, J. \& Dullemond, C. P. 2014, A\&A, 572, A78

Ercolano, B., Clarke, C. J., \& Drake, J. J. 2009, ApJ, 699, 1639

Ercolano, B., Drake, J. J., Raymond, J. C., \& Clarke, C. C. 2008, ApJ, 688, 398

Everett, J. E. 2005, ApJ, 631, 689

Fedele, D., van den Ancker, M. E., Henning, T., Jayawardhana,

R., \& Oliveira, J. M. 2010, A\&A, 510, A72

Fendt, C. \& Čemeljić, M. 2002, A\&A, 395, 1045

Ferreira, J. 1997, A\&A, 319, 340

Ferreira, J. \& Pelletier, G. 1995, A\&A, 295, 807

Flaherty, K. M., Hughes, A. M., Rosenfeld, K. A., Andrews, S. M., Chiang, E., Simon, J. B., Kerzner, S., \& Wilner, D. J. 2015, ApJ, 813, 99

Fleming, T. P., Stone, J. M., \& Hawley, J. F. 2000, ApJ, 530, 464 
Fogel, J. K. J., Bethell, T. J., Bergin, E. A., Calvet, N., \& Semenov, D. 2011, ApJ, 726, 29

Font, A. S., McCarthy, I. G., Johnstone, D., \& Ballantyne, D. R. 2004, ApJ, 607, 890

Fromang, S., Latter, H., Lesur, G., \& Ogilvie, G. I. 2013, A\&A, 552, A71

Fu, R. R., Weiss, B. P., Lima, E. A., Harrison, R. J., Bai, X.-N., Desch, S. J., Ebel, D. S., Suavet, C., Wang, H., Glenn, D., Le Sage, D., Kasama, T., Walsworth, R. L., \& Kuan, A. T. 2014, Science, 346, 1089

Gammie, C. F. 1996, ApJ, 457, 355

Garcia, P. J. V., Ferreira, J., Cabrit, S., \& Binette, L. 2001, A\&A, 377, 589

Glassgold, A. E., Najita, J., \& Igea, J. 2004, ApJ, 615, 972

Goodman, J. \& Pindor, B. 2000, Icarus, 148, 537

Gorti, U., Dullemond, C. P., \& Hollenbach, D. 2009, ApJ, 705, 1237

Gorti, U. \& Hollenbach, D. 2009, ApJ, 690, 1539

Gorti, U., Hollenbach, D., \& Dullemond, C. P. 2015, ApJ, 804, 29

Gressel, O., Turner, N. J., Nelson, R. P., \& McNally, C. P. 2015, ApJ, 801, 84

Guilet, J. \& Ogilvie, G. I. 2014, MNRAS, 441, 852

Gullbring, E., Hartmann, L., Briceno, C., \& Calvet, N. 1998, ApJ, 492, 323

Hartigan, P., Edwards, S., \& Ghandour, L. 1995, ApJ, 452, 736

Hartmann, L., Calvet, N., Gullbring, E., \& D'Alessio, P. 1998, ApJ, 495, 385

Hayashi, C. 1981, Progress of Theoretical Physics Supplement, 70,35

Hennebelle, P. \& Fromang, S. 2008, A\&A, 477, 9

Herczeg, G. J., Brown, J. M., van Dishoeck, E. F., \& Pontoppidan, K. M. 2011, A\&A, 533, A112

Hollenbach, D., Johnstone, D., Lizano, S., \& Shu, F. 1994, ApJ, 428,654

Igea, J. \& Glassgold, A. E. 1999, ApJ, 518, 848

Johansen, A., Oishi, J. S., Low, M.-M. M., Klahr, H., Henning, T., \& Youdin, A. 2007, Nature, 448, 1022

Johansen, A., Youdin, A., \& Mac Low, M. 2009, ApJ, 704, L75

Kato, S. X., Kudoh, T., \& Shibata, K. 2002, ApJ, 565, 1035

King, A. L., Miller, J. M., Raymond, J., Fabian, A. C., Reynolds, C. S., Kallman, T. R., Maitra, D., Cackett, E. M., \& Rupen, M. P. 2012, ApJ, 746, L20

Klaassen, P. D., Juhasz, A., Mathews, G. S., Mottram, J. C., De Gregorio-Monsalvo, I., van Dishoeck, E. F., Takahashi, S., Akiyama, E., Chapillon, E., Espada, D., Hales, A., Hogerheijde, M. R., Rawlings, M., Schmalzl, M., \& Testi, L. 2013, A\&A, 555, A73

Konigl, A. 1989, ApJ, 342, 208

Königl, A. 2004, ApJ, 617, 1267

Konigl, A. \& Kartje, J. F. 1994, ApJ, 434, 446

Königl, A., Salmeron, R., \& Wardle, M. 2010, MNRAS, 401, 479

Kraemer, S. B., George, I. M., Crenshaw, D. M., Gabel, J. R., Turner, T. J., Gull, T. R., Hutchings, J. B., Kriss, G. A., Mushotzky, R. F., Netzer, H., Peterson, B. M., \& Behar, E. 2005, ApJ, 633, 693

Krasnopolsky, R., Li, Z.-Y., \& Blandford, R. 1999, ApJ, 526, 631

Krasnopolsky, R., Li, Z.-Y., \& Blandford, R. D. 2003, ApJ, 595, 631

Kudoh, T. \& Shibata, K. 1997, ApJ, 474, 362

Lesur, G., Ferreira, J., \& Ogilvie, G. I. 2013, A\&A, 550, A61

Lesur, G., Kunz, M. W., \& Fromang, S. 2014, A\&A, 566, A56

Li, Z.-Y. 1995, ApJ, 444, 848

-. 1996, ApJ, 465, 855

Lovelace, R. V. E., Berk, H. L., \& Contopoulos, J. 1991, ApJ, 379,696

Lubow, S. H., Papaloizou, J. C. B., \& Pringle, J. E. 1994a, MNRAS, 267, 235

-. 1994b, MNRAS, 268, 1010

Lynden-Bell, D. 1996, MNRAS, 279, 389

-. 2003, MNRAS, 341, 1360

Machida, M. N., Inutsuka, S.-i., \& Matsumoto, T. 2008, ApJ, 676,1088

Mestel, L. 1961, MNRAS, 122, 473

Miller, J. M., Raymond, J., Fabian, A., Steeghs, D., Homan, J., Reynolds, C., van der Klis, M., \& Wijnands, R. 2006, Nature, 441, 953
Miller, J. M., Raymond, J., Fabian, A. C., Reynolds, C. S., King, A. L., Kallman, T. R., Cackett, E. M., van der Klis, M., \& Steeghs, D. T. H. 2012, ApJ, 759, L6

Mohanty, S., Ercolano, B., \& Turner, N. J. 2013, ApJ, 764, 65

Moll, R. 2012, A\&A, 548, A76

Murphy, G. C., Ferreira, J., \& Zanni, C. 2010, A\&A, 512, A82

Muzerolle, J., Hartmann, L., \& Calvet, N. 1998, AJ, 116, 455

Natta, A., Testi, L., Alcalá, J. M., Rigliaco, E., Covino, E.,

Stelzer, B., \& D'Elia, V. 2014, A\&A, 569, A5

Neilsen, J. \& Homan, J. 2012, ApJ, 750, 27

Neilsen, J. \& Lee, J. C. 2009, Nature, 458, 481

Nomura, H. \& Millar, T. J. 2005, A\&A, 438, 923

Ogilvie, G. I. 2012, MNRAS, 423, 1318

Oishi, J. S. \& Mac Low, M.-M. 2011, ApJ, 740, 18

Okuzumi, S., Takeuchi, T., \& Muto, T. 2014, ApJ, 785, 127

Ostriker, E. C. 1997, ApJ, 486, 291

Ouyed, R. \& Pudritz, R. E. 1997a, ApJ, 482, 712

-. 1997b, ApJ, 484, 794

-. 1999, MNRAS, 309, 233

Ouyed, R., Pudritz, R. E., \& Stone, J. M. 1997, Nature, 385, 409

Owen, J. E., Ercolano, B., Clarke, C. J., \& Alexander, R. D. 2010, MNRAS, 401, 1415

Panoglou, D., Cabrit, S., Pineau Des Forêts, G., Garcia, P. J. V., Ferreira, J., \& Casse, F. 2012, A\&A, 538, A2

Pascucci, I. \& Sterzik, M. 2009, ApJ, 702, 724

Pelletier, G. \& Pudritz, R. E. 1992, ApJ, 394, 117

Perez-Becker, D. \& Chiang, E. 2011, ApJ, 735, 8

Ponti, G., Fender, R. P., Begelman, M. C., Dunn, R. J. H.,

Neilsen, J., \& Coriat, M. 2012, MNRAS, 422, L11

Pontoppidan, K. M., Blake, G. A., \& Smette, A. 2011, ApJ, 733, 84

Porth, O. \& Fendt, C. 2010, ApJ, 709, 1100

Proga, D. 2007, in Astronomical Society of the Pacific Conference Series, Vol. 373, The Central Engine of Active Galactic Nuclei, ed. L. C. Ho \& J.-W. Wang, 267

Pudritz, R. E. \& Norman, C. A. 1983, ApJ, 274, 677

-. 1986, ApJ, 301, 571

Pudritz, R. E., Rogers, C. S., \& Ouyed, R. 2006, MNRAS, 365, 1131

Ramsey, J. P. \& Clarke, D. A. 2011, ApJ, 728, L11

Rigliaco, E., Pascucci, I., Gorti, U., Edwards, S., \& Hollenbach, D. 2013, ApJ, 772, 60

Riols, A., Rincon, F., Cossu, C., Lesur, G., Ogilvie, G. I., \& Longaretti, P.-Y. 2015, A\&A, 575, A14

Sacco, G. G., Flaccomio, E., Pascucci, I., Lahuis, F., Ercolano, B., Kastner, J. H., Micela, G., Stelzer, B., \& Sterzik, M. 2012, ApJ, 747, 142

Safier, P. N. 1993, ApJ, 408, 115

Salmeron, R., Königl, A., \& Wardle, M. 2011, MNRAS, 412, 1162

Salyk, C., Pontoppidan, K., Corder, S., Muñoz, D., Zhang, K., \& Blake, G. A. 2014, ApJ, 792, 68

Sheikhnezami, S. \& Fendt, C. 2015, ArXiv e-prints

Sheikhnezami, S., Fendt, C., Porth, O., Vaidya, B., \& Ghanbari, J. 2012, ApJ, 757, 65

Shu, F., Najita, J., Ostriker, E., Wilkin, F., Ruden, S., \& Lizano, S. 1994, ApJ, 429, 781

Shu, F. H., Lizano, S., Galli, D., Cai, M. J., \& Mohanty, S. 2008, ApJ, 682, L121

Sicilia-Aguilar, A., Henning, T., \& Hartmann, L. W. 2010, ApJ, 710,597

Simon, J. B., Bai, X.-N., Armitage, P. J., Stone, J. M., \& Beckwith, K. 2013a, ApJ, 775, 73

Simon, J. B., Bai, X.-N., Stone, J. M., Armitage, P. J., \& Beckwith, K. 2013b, ApJ, 764, 66

Simon, J. B., Lesur, G., Kunz, M. W., \& Armitage, P. J. 2015, MNRAS, 454, 1117

Spruit, H. C. 1996, in NATO ASIC Proc. 477: Evolutionary Processes in Binary Stars, ed. R. A. M. J. Wijers, M. B. Davies, \& C. A. Tout, 249-286

Stepanovs, D. \& Fendt, C. 2014, ApJ, 793, 31

Suzuki, T. K. \& Inutsuka, S.-i. 2009, ApJ, 691, L49

Teitler, S. 2011, ApJ, 733, 57

Tomida, K., Tomisaka, K., Matsumoto, T., Hori, Y., Okuzumi, S., Machida, M. N., \& Saigo, K. 2013, ApJ, 763, 6

Turner, N. J., Fromang, S., Gammie, C., Klahr, H., Lesur, G., Wardle, M., \& Bai, X.-N. 2014, Protostars and Planets VI, 411 
Tzeferacos, P., Ferrari, A., Mignone, A., Zanni, C., Bodo, G., \& Massaglia, S. 2009, MNRAS, 400, 820

-. 2013, MNRAS, 428, 3151

Ustyugova, G. V., Koldoba, A. V., Romanova, M. M., Chechetkin, V. M., \& Lovelace, R. V. E. 1999, ApJ, 516, 221

Vlahakis, N., Tsinganos, K., Sauty, C., \& Trussoni, E. 2000, MNRAS, 318, 417

Walsh, C., Millar, T. J., \& Nomura, H. 2010, ApJ, 722, 1607

Walsh, C., Nomura, H., Millar, T. J., \& Aikawa, Y. 2012, ApJ, 747,114

Wardle, M. 2007, Ap\&SS, 311, 35

Wardle, M. \& Koenigl, A. 1993, ApJ, 410, 218

Watson, D. M., Calvet, N. P., Fischer, W. J., Forrest, W. J., Manoj, P., Megeath, S. T., Melnick, G. J., Najita, J., Neufeld, D. A., Sheehan, P. D., Stutz, A. M., \& Tobin, J. J. 2015, ArXiv e-prints
Weber, E. J. \& Davis, Jr., L. 1967, ApJ, 148, 217

Weidenschilling, S. J. 1977, Ap\&SS, 51, 153

Williams, J. P. \& Best, W. M. J. 2014, ApJ, 788, 59

Woitke, P., Kamp, I., \& Thi, W.-F. 2009, A\&A, 501, 383

Youdin, A. N. \& Goodman, J. 2005, ApJ, 620, 459

Yuan, F., Bu, D., \& Wu, M. 2012, ApJ, 761, 130

Yuan, F., Gan, Z., Narayan, R., Sadowski, A., Bu, D., \& Bai, X.-N. 2015, ApJ, 804, 101

Zanni, C., Ferrari, A., Rosner, R., Bodo, G., \& Massaglia, S. 2007, A\&A, 469, 811 